\title{
HIV-1 Vpr Degrades TET2 to Suppress IRF7 and Interferon Expression in Plasmacytoid Dendritic Cells
}

Qi Wang ${ }^{1,2}$, Li-Chung Tsao ${ }^{1,2}$, Lei $\mathrm{Lv}^{1}$, Yanping Xu${ }^{1}$, Liang Cheng ${ }^{1,2}$, Haisheng Yu ${ }^{1,2}$,

Liguo Zhang ${ }^{1,3}$, Yue Xiong ${ }^{1}$ and Lishan $\mathrm{Su}^{1,2 *}$

${ }^{1}$ Lineberger Comprehensive Cancer Center, University of North Carolina at Chapel Hill, North

Carolina, USA.

${ }^{2}$ Department of Microbiology \& Immunology, University of North Carolina at Chapel Hill,

North Carolina, USA.

${ }^{3}$ IBP-CAS, Beijing, China.

*Correspondence: Lishan Su, lsu@ med.unc.edu

Running title: Vpr suppresses IFN-I induction in pDCs

Keywords: HIV-1, Vpr, TET2, pDC, IRF7, IFN-I 


\begin{abstract}
Plasmacytoid dendritic cells (pDCs) are the major source of type I interferons (IFN-I) in rapid response to viral infections, with constitutive expression of interferon regulatory factor 7 (IRF7). HIV-1 expresses several accessory proteins to counteract specific IFN-induced host restriction factors. As one abundant virion-associated protein, HIV-1 Vpr remains enigmatic in enhancing HIV-1 infection via unclear mechanisms. Here we report that Vpr impaired IFN-I induction in pDCs to enhance HIV-1 replication in CD4+ T cells. Blockade of IFN-I signaling abrogated the effect of Vpr on HIV-1 replication. Virion-associated Vpr suppressed IFN-I induction in pDC by TLR7 agonists. Modulation of IFN-I induction by Vpr was genetically dependent on its activity of TET2 degradation. We further demonstrate that Vpr-mediated TET2 degradation reduced expression of IRF7 in pDCs. Finally, degradation of TET2 in pDCs by Vpr reduced the demethylation level of the IRF7 promoter via CXXC5-dependent recruitment. We conclude that HIV-1 Vpr functions to promote HIV-1 replication by suppressing TET2-dependent IRF7 expression and IFN-I induction in pDCs. The Vpr-TET2-IRF7 axis provides a novel therapeutic target to control HIV-1 infection.
\end{abstract}




\section{Introduction}

During human immunodeficiency virus type 1 (HIV-1) infection, plasmacytoid dendritic cells (pDCs) are the major type I interferons (IFN-I) producer cells (Liu, 2005). The CD4+ pDCs are rapidly activated and are involved in initiating antiviral immunity and in suppressing HIV-1 replication.

To sense pathogen-associated molecule patterns (PAMPs) such as viral RNA or DNA, pDCs express the endosomal Toll-like receptors TLR7 and TLR9, which bind single-stranded RNA and CpG-containing DNA, respectively (Diebold et al., 2004; Hemmi et al., 2000). Activation of TLR7 or TLR9 leads to activation of MyD88 and subsequent phosphorylation and nuclear translocation of IFN regulatory factor 7 (IRF7) to bind the IFN-I promoter in pDCs (Guiducci et al., 2008; Honda et al., 2005b), which is critical for IFN-I induction in pDCs (Honda et al., 2005a; Smith et al., 2016). In contrast to other cell types, IRF7 is constitutively expressed in pDC and its activity in pDCs is regulated by both transcriptional and posttranscriptional mechanisms (Colina et al., 2008; Kim et al., 2016; Lee et al., 2013; Ma et al., 2017). Interestingly, the promoter of IRF7 contains methylated $\mathrm{CpG}$ islands that can be demethylated by the DNA demethylase TET2 via CXXC5-dependent recruitment (Ma et al., 2017).

Although HIV-1 can infect pDCs in humanized mice and human patients (Donaghy et al., 2003; Zhang et al., 2011), pDCs are not efficiently infected by HIV-1 in vitro. However, HIV-1 or HIVinfected cells can activate pDCs to produce IFN-I via CD4-dependent endocytosis without fusiondependent productive infection (Beignon et al., 2005). In spite of rapid induction of IFN-I, HIV-1 persists and establishes chronic infection in the infected hosts. To counteract IFN-induced antiviral immunity, HIV-1 expresses several accessory proteins to target specific IFN-stimulated genes (ISGs) or host restriction factors to maintain persistent replication. For example, HIV-1 Vif targets the ISGs APOBEC3 proteins to protect its genome integrity during replication and Vpu targets the 
ISG tetherin for degradation to allow release of HIV-1 virions from producer cells. The SIV Vpx is reported to target the SAMHD1 protein to facilitate SIV replication in macrophages and dendritic cells.

As one abundant virion-associated protein, however, HIV-1 Vpr remains enigmatic in enhancing HIV-1 infection via unclear mechanisms. It has been reported that, in nonhuman primates infected with Vpr mutant SIV, the Vpr mutations are reverted, resulting in a functional $V p r$ gene over the course of infection (Goh et al., 1998; Lang et al., 1993). Monkeys infected with a Vpr-null SIV mutant showed decreased virus replication and delayed disease progression (Gibbs et al., 1995; Hoch et al., 1995) and mutant Vpr in HIV-1 delayed viral replication in humanized mice (Sato et al., 2013). HIV-1 Vpr is a $14 \mathrm{kDa}$ protein that is efficiently packaged in HIV-1 virions (Selig et al., 1999), reportedly with 250-700 copies of Vpr proteins per HIV-1 particle (Muller et al., 2000). Upon HIV-1 entry, Vpr is released in target cells, which is implicated in enhancing early steps of viral replication and/or immune evasion. Biochemically, Vpr has been reported to interact with VprBP to mediate several key cellular activities including G2 cell cycle arrest (Wen et al., 2007). In a recent report, Vpr was postulated to cause G2 arrest and help escape immune sensing by activation of the SLX4 complex in target cells (Laguette et al., 2014), although SLX4-independent mechanisms are reported (Fregoso and Emerman, 2016; Zhou et al., 2016). In addition, Vpr has also been reported to target IRF3 for degradation in PM1 cells for immune evasion(Okumura et al., 2008) and to block the phosphorylation of TBK1 in myeloid cells to reduce IFN production (Harman et al., 2015). We have recently reported that Vpr/VprBP-CLR4 binds and degrades the DNA demethylase TET2 to enhance HIV-1 replication in macrophages via its effect on IL6 resolution(Lv et al., 2018) and IFITM3 expression(Wang and Su, 2019). The identification of the human hub silencing (HUSH) complex as a target for Vpr-mediated degradation also suggests that 
HIV-1 Vpr may alter chromatin remodeling complexes and host responses(Yurkovetskiy et al., 2018).

We extended our study and found that Vpr suppressed type 1 interferon induction in pDCs to enhance HIV-1 replication in co-cultured CD4+ T cells. In addition, we discovered that Vpr impaired IFN-I induction in pDCs through its binding with VprBP/TET2 degradation but independent of its G2 arrest activity. Lastly, Vpr led to reduced IRF7 expression by preventing its TET2-dependent demethylation. These findings reveal Vpr as the first HIV-1 protein that suppress host interferon induction and suggest targeting the Vpr-TET2-IRF7 axis in pDCs may prove clinically beneficial to control HIV-1 infection. 


\section{Results}

Vpr enhances HIV-1 replication in CD4 T cells co-cultured with pDCs by attenuating IFN-I induction in pDCs

CD4+ T cells are the major target cells for HIV-1 replication (Perelson et al., 1993), and Vpr doesn't affect HIV-1 replication in activated CD4+ T cells (Balliet et al., 1994; Planelles et al., 1995). Cell-free HIV-1 particles are poorly detected by pDCs, but HIV-1-infected CD4+ T cells are effectively sensed by pDCs to induce IFN-I expression (Colonna et al., 2004)'(Lepelley et al., 2011). HIV-1 or HIV-1 $\Delta$ Vpr infection in purified CD4+ T cells led to similar levels of HIV-1 infection (Figure 1A and S1). To determine if Vpr affects pDCs to modulate HIV-1 replication in CD4+ T cells, we co-cultured purified pDCs (Figure 1B and S1) with autologous CD4 T cells already infected with wild type HIV-1 or HIV-1 $1 \Delta$ Vpr viruses. As shown in the Figure 1C, p24 levels from HIV-1 or HIV-1 $\Delta \mathrm{Vpr}$ viruses-infected cells are comparable in the absence of pDCs. We also showed that, in the co-culture system, p24 was predominantly from infected CD4+ T cells but not pDCs (Figure S1C). However, CD4+ T cells infected with HIV-1 $\Delta$ Vpr mutant viruses were more sensitive to co-cultured pDCs (Fig. 1C/D). Summarized data from three different donors showed that Vpr could resist $\sim 50 \%$ of pDCs-mediated inhibition of viral replication. correlated with lower IFN- $\alpha$ induction (Fig. 1E). CD4+ T cells infected with HIV-1 or HIV-1 $\Delta$ Vpr viruses, or pDC alone, showed undetectable IFN- $\alpha$ induction (Fig. 1E). To determine whether the Vpr effect on HIV-1 replication was due to different IFN-I induction, we utilized an antibody against human IFN- $\alpha / \beta$ receptor 1 ( $\alpha$-IFNAR1) to specifically block IFN-I signaling (Cheng et al., 2017). Consistently, the IFNAR blocking mAb enhanced HIV-1 replication in CD4 T cells infected with HIV-1 or HIV-1 $\mathrm{Vpr}$ (Fig. 1F), and abrogated the Vpr activity to further enhance HIV-1 replication in CD4+ T cells cocultured with pDCs (Fig. 1F)(Kerkmann et al., 2003). 


\section{HIV-infected T cells activate pDC via CD4-dependent but RT-independent mechanisms.}

To define how HIV-infected T cells activate pDC in the coculture model, we found that sCD4 blocked IFN-I induction in a dose-dependent manner (Fig. 2A). Consistent with the IFNAR blockade results (Fig. 1F), sCD4 also elevated HIV-1 replication in the co-culture model and abrogated the Vpr activity to further enhance HIV-1 replication (Fig. 2B). In contrast, inhibition of RT (reverse transcription) in the coculture model showed no effect on IFN-I induction by T cells with established infection of either Vpr+ or Vpr- viruses (Fig. 2C). Consistent with predominantly established infection in T cells, RT inhibition showed only minor effect on HIV-1 replication, probably due to low levels of new infection (Fig. 2D).

\section{Virion-associated Vpr modulates IFN-I induction in pDCs via its TET2 degradation activity.}

HIV-1 activates pDCs through endosomal recognition of viral RNA by TLR7(Beignon et al., 2005; Lepelley et al., 2011). To test how Vpr in HIV-1 particles affects activation of pDCs, we utilized the TLR7/8 agonist, R848, to activate pDCs in the presence of HIV-1 or HIV-1 $\mathrm{V}$ pr viruses. As shown in Figure 3A, cell-free HIV-1 alone at the used dose failed to activate pDCs as previously reported (Bloch et al., 2014; Su et al., 2014). However, wild type HIV-1 but not HIV-1 $\Delta$ Vpr viruses significantly reduced IFN- $\alpha$ induction by R848. To confirm that virion-associated Vpr was sufficient to suppress IFN-I production in pDC during R848 treatment, we utilized virus-like particles containing Vpr (VLPVPR) or no Vpr (VLP) to treat pDCs (Fig. S2A). Similar to cell-free HIV-1 viruses, VLP +/- Vpr failed to activate $\mathrm{pDC}$ and $\mathrm{pDC}$ treated with VLPvPR showed significantly reduced IFN-I expression in response to R848 (Figure 3B). When the kinetics of IFNI mRNA induction was measured (Figure S2B), VLPvPR impaired IFN-I expression during the early induction phase in response to R848. Virion-associated Vpr thus contributes to the reduced IFN-I induction in pDCs. 
Among a range of functions of the Vpr protein, Vpr-binding to VprBP and Vpr-dependent G2 arrest are the most extensively explored features (Belzile et al., 2007). We used Vpr mutants with specific defective activities of Vpr to define the Vpr-mediated impairment of IFN-I production in pDCs. Both VprQ65R and Vpre80A mutants are defective for induction of G2/M arrest (Belzile et al., 2007). VprQ65R, but not $\operatorname{Vpr}_{\text {R80A }}$ mutant, is defective for binding with VprBP (Le Rouzic et al., 2007). We recently discovered that HIV-1 Vpr targets the Tet methylcytosine dioxygenase 2 (TET2), a methylcytosine dioxygenase, via VprBP for degradation(Lv et al., 2018; Wang and Su, 2019). We produced virus-like particles containing VprwT, VprQ65R or VprR80A and all three Vpr proteins were efficiently incorporated into VLP (Figure S2B and(Lv et al., 2018; Wang and Su, 2019)). Similar to the Vpr activity in suppressing IFN-I expression in pDCs, VLPvPR-wT and VLPVPR-R80A but not VLP and VLPVPR-Q65R degraded TET2 proteins in pDCs (Figure 3C and Fig. S3). VLPVPR-wT and VLPVPR-R80A showed similar TET2 degradation (Fig. S3A) and inhibition of IFN-I induction in pDCs as measured by IFN- $\alpha$ protein (Figure $3 \mathrm{C}$ ) or mRNA levels of IFN- $\alpha$ and IFN- $\beta$ (Figure S3B), whereas VLPVPR-Q65R (like VLP with no Vpr) showed no activity. When TET2 was depleted by shRNA (Fig. S3C), induction of IFN-I by R848 was reduced by > 2-fold and Vpr failed to further reduce its induction (Fig. 3D). Therefore, Vpr interaction with VprBP to degrade TET2, but not its G2 cell cycle arrest activity, is critical for the Vpr-mediated inhibition of pDC activation.

\section{Vpr reduces IRF7 expression in pDCs}

To determine if Vpr altered expression of the critical signaling molecules involved in IFN-I expression in pDCs, we measured their protein levels and discovered that IRF7 protein levels, but not other molecules in the pathway, were reduced by $\sim 40 \%$ in the presence of VLPVPR-WT and VLPvPr-R80A (Fig. 4A). We further showed that VLPVPR-wT and VLPVPR-R80A, but not VLP or VLPVPR-Q65R, reduced IRF7 mRNA levels in pDCs (Fig. 4B). When IRF7 mRNA was measured 
in control or TET2-KD pDC, TET2-KD reduced IRF7 expression and abrogated the Vpr activity to further reduce IRF7 expression (Fig. 4C). These results suggest that Vpr degrades TET2 to reduce IRF7 gene expression in pDCs to modulate IFN-I induction.

\section{Vpr degrades TET2 to prevent demethylation of the IRF7 promoter in pDCs}

TET2 catalyzes the conversion of 5-methylcytosine $(5 \mathrm{mC})$ to 5-hydroxymethylcytosine (5hmC), leading to DNA demethylation to enhance gene expression (Abdel-Wahab et al., 2009). We showed that the pDC-specific CXXC5 protein recruited TET2 to the IRF7 promoter (Fig. 5A-C). To determine the $5 \mathrm{hmC}$ level on the IRF7 promoter in pDCs, we performed the anti-5hmC ChIPqPCR assay on the IRF7 promoter and showed TET2 depended on CXXC5 to demethylate 5mC to $5 \mathrm{hmC}$ at the $\mathrm{CpG}$ island (Fig. 5D). Interestingly, correlated with their ability to degrade TET2, VLPVPR-wT and VLPVPR-R80A but not VLPVPR-Q65R substantially reduced 5hmC levels at the IRF7 promoter in pDCs (Fig. 5E). These results demonstrate that HIV-1 Vpr reduced IRF7 and IFN-I gene expression through reducing TET2-dependent demethylation of the IRF7 promoter (Fig. 5F). 


\section{Discussion}

Vpr is a highly conserved HIV-1 protein which is associated with high viral load and required for full pathogenesis by unclear mechanisms (Gibbs et al., 1995; Goh et al., 1998). We report here that Vpr promoted HIV-1 replication in CD4+ T cells when co-cultured with pDCs via IFN-I signaling dependent mechanisms. HIV-1 Vpr impaired IFN-I production in pDCs to confer partial resistance of HIV-infected-CD4+ T cells to resist viral inhibition by pDCs. Moreover, we showed that Vpr reduced IRF7 transcription and IFN-I production through degradation of TET2 in pDCs (Figure 5).

In agreement with a lack of IFN-I induction in T cells, Vpr didn't affect HIV-1 replication in purified and activated CD4+ T cells (Figure S1B)(Balliet et al., 1994; Planelles et al., 1995). However, Vpr conferred HIV-infected CD4+ T cells to partially resist co-cultured autologous pDCs (Figure 1). As professional IFN-I-producing cells, pDCs rapidly produced high levels of IFN-I upon exposure to HIV-infected CD4+ T cells. We blocked IFN-I signaling and found HIV1 replication were elevated to the same level in the presence or absence of Vpr when HIV-infected CD4+ T cells were co-cultured with pDCs (Fig. 1F), indicating that the major Vpr effect on pDCmediated suppression of HIV-1 in T cells was due to differential induction of IFN-I. Interaction of HIV-infected T cells with CD4 was critical for the induction of IFN-I in pDC, although inhibition of reverse transcription showed no effect. Together the data indicate that pDC efficiently sensed HIV-infected T cells via CD4-depedent mechanism to induce IFN-I, which was impaired by Vpr.

IFN-I induces a number of anti-viral factors to inhibit HIV-1 replication in target cells (Kane et al., 2016), including APOBEC3, SAMHD1 and Mx2 (Hatziioannou and Bieniasz, 2011; Hotter and Kirchhoff, 2018). The results presented here showing that Vpr impaired IFN-I production in pDCs may partly account for Vpr-mediated enhancement of viral replication in SIVinfected monkeys and in HIV-1 infected humanized mice (Gibbs et al., 1995; Hoch et al., 1995)'(Sato et al., 2013). Therefore, HIV-1 seems to have evolved to efficiently activate pDC via 
CD4-dependent endocytosis and TLR7 signaling. However, the magnitude of IFN-I induction is modulated by the Vpr protein to a low level that other HIV-1 accessory proteins are able to counteract the specific anti-HIV ISG restriction factors to maintain HIV-1 infection.

It is reported that Vpr may have a positive effect on IFN-I induction during HIV-1 infection of monocytes-derived DC (MDDCs) or monocytes-derived macrophages (MDMs) (Zahoor et al., 2015; Zahoor et al., 2014). In contrast, others have reported a suppressive role of Vpr on IFN-I production in MDDCs and MDMs (Harman et al., 2011; Mashiba et al., 2014), or in cell lines, such as HEK293, HeLa and HeLa-derived STING-expressing reporter cells (Vermeire et al., 2016).

Compared to conventional dendritic cells and macrophages, human pDCs express only the endoplasmic TLR7/9 but not other pattern recognition receptors (PRR) (Colonna et al., 2004). Once pDCs sense pathogens through TLR7/9, signaling is mediated via MyD88 (myeloid differentiation factor 88), a docking protein for IRAK1/4 (IL-1R-associated kinase 1/4). IFNregulatory factor 7 (IRF7) is then phosphorylated and translocated into the nucleus to promote IFN-I gene expression (Honda et al., 2005b). It is well documented that pDCs uniquely express constitutive levels of IRF7 involving reduced methylation levels at the IRF7 promoter (Kulaeva et al., 2003). We further discovered that Vpr degraded TET2 through the CRL4 ${ }^{\mathrm{VprBP}}$ E3 ligase ligase (Lv et al., 2018) to suppress the IRF7 gene expression (Figure 4). CpG island on the human IRF7 promoter has been located around the TATA box spanning from -271 to +382 bp (Lu et al., 2000). We showed that Vpr-treated pDCs (or with TET2 shRNA) had reduced 5hmc levels at the IRF7 promoter (Figure 5E), associated with reduced IRF7 levels. Since TET2 does not have a specific DNA binding domain, we also showed that the pDC-specific factor CXXC5 was involved to recruit TET2 to demethylate the IRF7 promoter (Fig. 5 and (Ma et al., 2017)). It is likely that TET2 also demethylate other genes recruited by CXXC5 or other factors. Other putative target genes affected by Vpr or depletion of TET2 in pDCs are of great interest and will be studied in the future. 
In summary, we have discovered that Vpr targets the TET2-IRF7 axis to modulate IFN-I induction in pDCs, leading to elevated HIV-1 replication in HIV-infected T cells. This Vpr activity depended on its interaction with the VprBP-CRL4 complex to degrade TET2. Interestingly, The constitutive expression of IRF7 and CXXC5 in pDCs is maintained by TET2-mediated demethylation of the IRF7 promoter. The dissection of the molecular mechanisms modulating IFN-I induction in pDCs by Vpr during HIV-1 infection could uncover new targets or strategy to treat HIV-1 replication and pathogenesis. 


\section{Materials and Methods}

\section{Viruses and plasmids}

pNL4-3-R+ and pNL4-3-R- were obtained from the NIH AIDS Reagent program (Adachi et al., 1986; Connor et al., 1995; He et al., 1995). Flag-VprQ65R and Flag-VprR80A were generated by sitedirected mutagenesis and verified by DNA sequencing.

\section{VLP stock production and transduction}

For the production of virus-like-particles (VLP) carrying Vpr or Vpr-mutants, we transfected 293T with $6 \mu \mathrm{g}$ p3 x FLAG-CMV-Vpr, $15 \mu \mathrm{g}$ pMDLg/pRRE, $4 \mu \mathrm{g}$ pRSV-Rev, and $5 \mu \mathrm{g}$ pCMV-VSVG. The VLP stock was treated with DNase (25 U DNase per $0.5 \mathrm{ml}$ virus) before transduction. Concentration of VLP stocks were quantified by p24 ELISA assay (Frederick National Laboratory for Cancer Research - AIDS and Cancer Virus Program). All VLPs of 500 ng p24 amount and virus infections were performed by spin inoculation for 2 hours at $1500 \mathrm{~g}$ and $37^{\circ} \mathrm{C}$ with $8 \mu \mathrm{g} / \mathrm{ml}$ polybrene (O'Doherty et al., 2000).

\section{Cell cultures and stimulation}

pDC-like Gen2.2 cells (Chaperot et al., 2001) were kept as stock in our lab. Gen2.2 cells were maintained in RPMI 1640 medium containing 10\% FBS, 1\% Pen/Strep antibiotics, 2mM glutamine, 10mM HEPES, 1x nonessential amino acids and 1x Pyruvate (all from GIBCO). 293T cells were cultured in DMEM containing 10\% FBS and 1X Antibiotic-Antimycotic (Invitrogen). Human buffy coats were obtained from the Gulf Coast Regional Blood Center. Primary human peripheral blood mononuclear cells (PBMCs) were isolated from buffy coats using Ficoll-paque gradient. BDCA-4 Microbead Kit (Miltenyi Biotec) was used to isolate human pDCs from PBMCs. Primary CD4+ T cells were negatively isolated from PBMCs using untouched dynabeads human CD4 T cell kit (Invitrogen, CAT\# 11352D) according to manufacturer's manual. Isolated CD4 T cells were stimulated with anti-CD3/anti-CD28 coated magnetic beads (Gibco, CAT\#11131D) at bead:cell ratio 1:1, and maintained in RPMI 1640 (Invitrogen) supplemented with 10\% fetal 
bovine serum (FBS) plus 200 IU/ml human recombinant IL-2 (NIH AIDS Reagent Program). Beads were removed at day 3 after the stimulation. $1 \mu \mathrm{g} / \mathrm{ml}$ R848 (Invivogen, tlrl-r848), $1 \mu \mathrm{m}$ CpGA (Invivogen, tlrl-2216) and $1 \mu \mathrm{m}$ CpG-B (Invivogen, tlrl-2006) are used to activated pDCs. 10 $\mu \mathrm{g} / \mathrm{ml} \mathrm{R} 848$ was used to activate Gen2.2 cells.

\section{ShRNA and RNA quantitation by real-time PCR}

pGIPZ-lentiviral vectors expressing control (non-targeting), TET2-targeting shRNA were purchased from Dharmacon, GE Healthcare (Lafayette, CO). shRNA targeting human TET2 has mature antisense sequence: TAAGTAATACAATGTTCTT (clone ID: V3LHS_363201). Lentiviruses for shRNA targeting TET2 were produced by $\mathrm{CaCl} 2-\mathrm{BES}$ transfection of $293 \mathrm{~T}$ cells (10 cm plate) using $10 \mathrm{ug}$ vector, $15 \mu \mathrm{g}$ pMDLg/pRRE, $4 \mu \mathrm{g}$ pRSV-Rev, and $5 \mu \mathrm{g}$ pCMV-VSVG. Gen2.2 cells were transduced with $200 \mathrm{ng}$ p24 of lentiviruses, and after 3 days sorted by GFP+ by flow cytometry. The recovered GFP+ cells were validated by western blot. For detection IFN-I and IRF7 expression. The primers are as following. IFN-a, F: GCCTCGCCCTTtGCTTtACT, R: CTGTGGGTCTCAGGGAGATCA. IFN-b, F: GTGCCTGGACCATAGTCAGAGTGG， R: TGTCCAGTCCCAGAGGCACAGG. IRF7，F: ACAGCACAGGGC GTtTtATC, R: TCCCGGCTAAGTTCGTACAC. GAPDH, F: CATGAGAAGTATGACAACAGCCT, R: AGTCCTTCCACGATACCAAAGT. Real-time PCR was performed using the SYBR green fluorescence system. IRF7 CHIP primer, F: GGGTTCCGCTGGTCGCATCC, R: CCCGCCCGACCCTCATCTCT.

\section{ELISA}

ELISA kits for human IFN- $\alpha$ (Mabtech; 3425-1H-20) were used according to the manufacturer's instructions. ELISA kits for p24 (Leidos, Biomedical Research Inc.) were used according to the instructions of the manufacturer.

\section{Antibodies}


PerCP-Cy5-5-conjugated CD123 (Biolegend), FITC-conjugated DR (Biolegend), FITCconjugated CD3 (Biolegend), APC-Cy7-conjugated CD4 (Biolegend), anti-5hmc (ACTIVE MOTIF) and anti-TET2 was obtained from Millipore (MABE462).

\section{Statistical analysis}

All statistical analyses were performed with the unpaired Student's t-test, and were considered significant when the $\mathrm{p}$-value was less than $0.05 . *, * *$ and $* * *$ indicate respectively $\mathrm{p}$-values of less than $0.05,0.01$ and 0.001 . 


\section{Authorship contributions}

Q.W. and L.T. designed and performed experiments, and analyzed the results and wrote the paper; L.L, Y.Z, L.C., H.Y. performed experiments and analyzed data; L.Z. and Y. X. provided reagents and analyzed data; L.S. conceived the research project, planned, designed the experiments, analyzed results and wrote the paper.

\section{Acknowledgements}

We thank Liqun Chi and Yaxu Wu for technical assistance. We also thank the University of North Carolina (UNC) Division of Laboratory Medicine for animal care; University of North Carolina at Chapel Hill Center for AIDS Research (P30 AI50410), Flow Cytometry Core Facility, and the UNC Animal Histopathology Core Facility. We thank all members in the Su laboratory for critical reading and/or discussion of the manuscript.

We thank the NIH AIDS Research and Reference Reagent Program for kindly providing reagents. We thank Amanda Pons Taylor from Lineberger Comprehensive Cancer Center at the University of North Carolina at Chapel Hill for reading the manuscript. This study was supported in part by NIH grants AI095097 (to L.S.), CA163834 (to Y.X.) and AI127346 to L.S. and Y.X. The funders had no role in data analysis, preparation, or decision to publish the manuscript. 


\section{References}

Abdel-Wahab, O., A. Mullally, C. Hedvat, G. Garcia-Manero, J. Patel, M. Wadleigh, S. Malinge, J. Yao, O. Kilpivaara, R. Bhat, K. Huberman, S. Thomas, I. Dolgalev, A. Heguy, E. Paietta, M.M. Le Beau, M. Beran, M.S. Tallman, B.L. Ebert, H.M. Kantarjian, R.M. Stone, D.G. Gilliland, J.D. Crispino, and R.L. Levine. 2009. Genetic characterization of TET1, TET2, and TET3 alterations in myeloid malignancies. Blood 114:144-147.

Adachi, A., H.E. Gendelman, S. Koenig, T. Folks, R. Willey, A. Rabson, and M.A. Martin. 1986. Production of acquired immunodeficiency syndrome-associated retrovirus in human and nonhuman cells transfected with an infectious molecular clone. Journal of virology 59:284-291.

Balliet, J.W., D.L. Kolson, G. Eiger, F.M. Kim, K.A. McGann, A. Srinivasan, and R. Collman. 1994. Distinct effects in primary macrophages and lymphocytes of the human immunodeficiency virus type 1 accessory genes vpr, vpu, and nef: mutational analysis of a primary HIV-1 isolate. Virology 200:623631.

Beignon, A.S., K. McKenna, M. Skoberne, O. Manches, I. DaSilva, D.G. Kavanagh, M. Larsson, R.J. Gorelick, J.D. Lifson, and N. Bhardwaj. 2005. Endocytosis of HIV-1 activates plasmacytoid dendritic cells via Toll-like receptor-viral RNA interactions. The Journal of clinical investigation 115:3265-3275.

Belzile, J.P., G. Duisit, N. Rougeau, J. Mercier, A. Finzi, and E.A. Cohen. 2007. HIV-1 Vpr-mediated G2 arrest involves the DDB1-CUL4AVPRBP E3 ubiquitin ligase. PLoS pathogens 3:e85.

Bloch, N., M. O'Brien, T.D. Norton, S.B. Polsky, N. Bhardwaj, and N.R. Landau. 2014. HIV type 1 infection of plasmacytoid and myeloid dendritic cells is restricted by high levels of SAMHD1 and cannot be counteracted by Vpx. AIDS research and human retroviruses 30:195-203.

Chaperot, L., N. Bendriss, O. Manches, R. Gressin, M. Maynadie, F. Trimoreau, H. Orfeuvre, B. Corront, J. Feuillard, J.J. Sotto, J.C. Bensa, F. Briere, J. Plumas, and M.C. Jacob. 2001. Identification of a leukemic counterpart of the plasmacytoid dendritic cells. Blood 97:3210-3217.

Cheng, L., J. Ma, J. Li, D. Li, G. Li, F. Li, Q. Zhang, H. Yu, F. Yasui, C. Ye, L.C. Tsao, Z. Hu, L. Su, and L. Zhang. 2017. Blocking type I interferon signaling enhances T cell recovery and reduces HIV-1 reservoirs. The Journal of clinical investigation 127:269-279.

Colina, R., M. Costa-Mattioli, R.J. Dowling, M. Jaramillo, L.H. Tai, C.J. Breitbach, Y. Martineau, O. Larsson, L. Rong, Y.V. Svitkin, A.P. Makrigiannis, J.C. Bell, and N. Sonenberg. 2008. Translational control of the innate immune response through IRF-7. Nature 452:323-328.

Colonna, M., G. Trinchieri, and Y.J. Liu. 2004. Plasmacytoid dendritic cells in immunity. Nature immunology 5:1219-1226.

Connor, R.I., B.K. Chen, S. Choe, and N.R. Landau. 1995. Vpr is required for efficient replication of human immunodeficiency virus type-1 in mononuclear phagocytes. Virology 206:935-944.

Diebold, S.S., T. Kaisho, H. Hemmi, S. Akira, and C. Reis e Sousa. 2004. Innate antiviral responses by means of TLR7-mediated recognition of single-stranded RNA. Science 303:1529-1531.

Donaghy, H., B. Gazzard, F. Gotch, and S. Patterson. 2003. Dysfunction and infection of freshly isolated blood myeloid and plasmacytoid dendritic cells in patients infected with HIV-1. Blood 101:45054511.

Fregoso, O.I., and M. Emerman. 2016. Activation of the DNA Damage Response Is a Conserved Function of HIV-1 and HIV-2 Vpr That Is Independent of SLX4 Recruitment. MBio 7:

Gibbs, J.S., A.A. Lackner, S.M. Lang, M.A. Simon, P.K. Sehgal, M.D. Daniel, and R.C. Desrosiers. 1995. Progression to AIDS in the absence of a gene for vpr or vpx. Journal of virology 69:2378-2383.

Goh, W.C., M.E. Rogel, C.M. Kinsey, S.F. Michael, P.N. Fultz, M.A. Nowak, B.H. Hahn, and M. Emerman. 1998. HIV-1 Vpr increases viral expression by manipulation of the cell cycle: a mechanism for selection of Vpr in vivo. Nature medicine 4:65-71.

Guiducci, C., C. Ghirelli, M.A. Marloie-Provost, T. Matray, R.L. Coffman, Y.J. Liu, F.J. Barrat, and V. Soumelis. 2008. PI3K is critical for the nuclear translocation of IRF-7 and type I IFN production by 
human plasmacytoid predendritic cells in response to TLR activation. The Journal of experimental medicine 205:315-322.

Harman, A.N., J. Lai, S. Turville, S. Samarajiwa, L. Gray, V. Marsden, S.K. Mercier, K. Jones, N. Nasr, A. Rustagi, H. Cumming, H. Donaghy, J. Mak, M. Gale, Jr., M. Churchill, P. Hertzog, and A.L. Cunningham. 2011. HIV infection of dendritic cells subverts the IFN induction pathway via IRF-1 and inhibits type 1 IFN production. Blood 118:298-308.

Harman, A.N., N. Nasr, A. Feetham, A. Galoyan, A.A. Alshehri, D. Rambukwelle, R.A. Botting, B.M. Hiener, E. Diefenbach, R.J. Diefenbach, M. Kim, A. Mansell, and A.L. Cunningham. 2015. HIV Blocks Interferon Induction in Human Dendritic Cells and Macrophages by Dysregulation of TBK1. Journal of virology 89:6575-6584.

Hatziioannou, T., and P.D. Bieniasz. 2011. Antiretroviral restriction factors. Current opinion in virology 1:526-532.

He, J., S. Choe, R. Walker, P. Di Marzio, D.O. Morgan, and N.R. Landau. 1995. Human immunodeficiency virus type 1 viral protein $R(\mathrm{Vpr})$ arrests cells in the $\mathrm{G} 2$ phase of the cell cycle by inhibiting p34cdc2 activity. Journal of virology 69:6705-6711.

Hemmi, H., O. Takeuchi, T. Kawai, T. Kaisho, S. Sato, H. Sanjo, M. Matsumoto, K. Hoshino, H. Wagner, K. Takeda, and S. Akira. 2000. A Toll-like receptor recognizes bacterial DNA. Nature 408:740-745.

Hoch, J., S.M. Lang, M. Weeger, C. Stahl-Hennig, C. Coulibaly, U. Dittmer, G. Hunsmann, D. Fuchs, J. Muller, S. Sopper, and et al. 1995. vpr deletion mutant of simian immunodeficiency virus induces AIDS in rhesus monkeys. Journal of virology 69:4807-4813.

Honda, K., Y. Ohba, H. Yanai, H. Negishi, T. Mizutani, A. Takaoka, C. Taya, and T. Taniguchi. 2005a. Spatiotemporal regulation of MyD88-IRF-7 signalling for robust type-I interferon induction. Nature 434:1035-1040.

Honda, K., H. Yanai, H. Negishi, M. Asagiri, M. Sato, T. Mizutani, N. Shimada, Y. Ohba, A. Takaoka, N. Yoshida, and T. Taniguchi. 2005b. IRF-7 is the master regulator of type-I interferon-dependent immune responses. Nature 434:772-777.

Hotter, D., and F. Kirchhoff. 2018. Interferons and beyond: Induction of antiretroviral restriction factors. Journal of leukocyte biology 103:465-477.

Kane, M., T.M. Zang, S.J. Rihn, F. Zhang, T. Kueck, M. Alim, J. Schoggins, C.M. Rice, S.J. Wilson, and P.D. Bieniasz. 2016. Identification of Interferon-Stimulated Genes with Antiretroviral Activity. Cell host \& microbe 20:392-405.

Kerkmann, M., S. Rothenfusser, V. Hornung, A. Towarowski, M. Wagner, A. Sarris, T. Giese, S. Endres, and G. Hartmann. 2003. Activation with CpG-A and CpG-B oligonucleotides reveals two distinct regulatory pathways of type I IFN synthesis in human plasmacytoid dendritic cells. Journal of immunology 170:4465-4474.

Kim, T.W., S. Hong, Y. Lin, E. Murat, H. Joo, T. Kim, V. Pascual, and Y.J. Liu. 2016. Transcriptional Repression of IFN Regulatory Factor 7 by MYC Is Critical for Type I IFN Production in Human Plasmacytoid Dendritic Cells. Journal of immunology 197:3348-3359.

Kulaeva, O.I., S. Draghici, L. Tang, J.M. Kraniak, S.J. Land, and M.A. Tainsky. 2003. Epigenetic silencing of multiple interferon pathway genes after cellular immortalization. Oncogene 22:4118-4127.

Laguette, N., C. Bregnard, P. Hue, J. Basbous, A. Yatim, M. Larroque, F. Kirchhoff, A. Constantinou, B. Sobhian, and M. Benkirane. 2014. Premature activation of the SLX4 complex by Vpr promotes G2/M arrest and escape from innate immune sensing. Cell 156:134-145.

Lang, S.M., M. Weeger, C. Stahl-Hennig, C. Coulibaly, G. Hunsmann, J. Muller, H. Muller-Hermelink, D. Fuchs, H. Wachter, M.M. Daniel, and et al. 1993. Importance of vpr for infection of rhesus monkeys with simian immunodeficiency virus. Journal of virology 67:902-912.

Le Rouzic, E., N. Belaidouni, E. Estrabaud, M. Morel, J.C. Rain, C. Transy, and F. Margottin-Goguet. 2007. HIV1 Vpr arrests the cell cycle by recruiting DCAF1/VprBP, a receptor of the Cul4-DDB1 ubiquitin ligase. Cell Cycle 6:182-188. 
Lee, M.S., B. Kim, G.T. Oh, and Y.J. Kim. 2013. OASL1 inhibits translation of the type I interferonregulating transcription factor IRF7. Nature immunology 14:346-355.

Lepelley, A., S. Louis, M. Sourisseau, H.K. Law, J. Pothlichet, C. Schilte, L. Chaperot, J. Plumas, R.E. Randall, M. Si-Tahar, F. Mammano, M.L. Albert, and O. Schwartz. 2011. Innate sensing of HIVinfected cells. PLoS pathogens 7:e1001284.

Liu, Y.J. 2005. IPC: professional type 1 interferon-producing cells and plasmacytoid dendritic cell precursors. Annual review of immunology 23:275-306.

Lu, R., W.C. Au, W.S. Yeow, N. Hageman, and P.M. Pitha. 2000. Regulation of the promoter activity of interferon regulatory factor- 7 gene. Activation by interferon snd silencing by hypermethylation. The Journal of biological chemistry 275:31805-31812.

Lv, L., Q. Wang, Y. Xu, L.C. Tsao, T. Nakagawa, H. Guo, L. Su, and Y. Xiong. 2018. Vpr Targets TET2 for Degradation by CRL4(VprBP) E3 Ligase to Sustain IL-6 Expression and Enhance HIV-1 Replication. Mol Cell 70:961-970 e965.

Ma, S., X. Wan, Z. Deng, L. Shi, C. Hao, Z. Zhou, C. Zhou, Y. Fang, J. Liu, J. Yang, X. Chen, T. Li, A. Zang, S. Yin, B. Li, J. Plumas, L. Chaperot, X. Zhang, G. Xu, L. Jiang, N. Shen, S. Xiong, X. Gao, Y. Zhang, and H. Xiao. 2017. Epigenetic regulator CXXC5 recruits DNA demethylase Tet2 to regulate TLR7/9-elicited IFN response in pDCs. The Journal of experimental medicine 214:1471-1491.

Mashiba, M., D.R. Collins, V.H. Terry, and K.L. Collins. 2014. Vpr overcomes macrophage-specific restriction of HIV-1 Env expression and virion production. Cell host \& microbe 16:722-735.

Muller, B., U. Tessmer, U. Schubert, and H.G. Krausslich. 2000. Human immunodeficiency virus type 1 Vpr protein is incorporated into the virion in significantly smaller amounts than gag and is phosphorylated in infected cells. Journal of virology 74:9727-9731.

O'Doherty, U., W.J. Swiggard, and M.H. Malim. 2000. Human immunodeficiency virus type 1 spinoculation enhances infection through virus binding. Journal of virology 74:10074-10080.

Okumura, A., T. Alce, B. Lubyova, H. Ezelle, K. Strebel, and P.M. Pitha. 2008. HIV-1 accessory proteins VPR and Vif modulate antiviral response by targeting IRF-3 for degradation. Virology 373:85-97.

Perelson, A.S., D.E. Kirschner, and R. De Boer. 1993. Dynamics of HIV infection of CD4+ T cells. Mathematical biosciences 114:81-125.

Planelles, V., F. Bachelerie, J.B. Jowett, A. Haislip, Y. Xie, P. Banooni, T. Masuda, and I.S. Chen. 1995. Fate of the human immunodeficiency virus type 1 provirus in infected cells: a role for vpr. Journal of virology 69:5883-5889.

Sato, K., N. Misawa, S. Iwami, Y. Satou, M. Matsuoka, Y. Ishizaka, M. Ito, K. Aihara, D.S. An, and Y. Koyanagi. 2013. HIV-1 Vpr accelerates viral replication during acute infection by exploitation of proliferating CD4+ T cells in vivo. PLoS pathogens 9:e1003812.

Selig, L., J.C. Pages, V. Tanchou, S. Preveral, C. Berlioz-Torrent, L.X. Liu, L. Erdtmann, J. Darlix, R. Benarous, and S. Benichou. 1999. Interaction with the $\mathrm{p} 6$ domain of the gag precursor mediates incorporation into virions of $\mathrm{Vpr}$ and $\mathrm{Vpx}$ proteins from primate lentiviruses. Journal of virology 73:592-600.

Smith, N., P.O. Vidalain, S. Nisole, and J.P. Herbeuval. 2016. An efficient method for gene silencing in human primary plasmacytoid dendritic cells: silencing of the TLR7/IRF-7 pathway as a proof of concept. Scientific reports 6:29891.

Su, B., A. Lederle, G. Laumond, C. Ducloy, S. Schmidt, T. Decoville, and C. Moog. 2014. Broadly neutralizing antibody VRCO1 prevents HIV-1 transmission from plasmacytoid dendritic cells to CD4 T lymphocytes. Journal of virology 88:10975-10981.

Vermeire, J., F. Roesch, D. Sauter, R. Rua, D. Hotter, A. Van Nuffel, H. Vanderstraeten, E. Naessens, V. Iannucci, A. Landi, W. Witkowski, A. Baeyens, F. Kirchhoff, and B. Verhasselt. 2016. HIV Triggers a cGAS-Dependent, Vpu- and Vpr-Regulated Type I Interferon Response in CD4(+) T Cells. Cell reports 17:413-424.

Wang, Q., and L. Su. 2019. Vpr Enhances HIV-1 Env Processing and Virion Infectivity in Macrophages by Modulating TET2-Dependent IFITM3 Expression. MBio 10: 
Wen, X., K.M. Duus, T.D. Friedrich, and C.M. de Noronha. 2007. The HIV1 protein Vpr acts to promote G2 cell cycle arrest by engaging a DDB1 and Cullin4A-containing ubiquitin ligase complex using VprBP/DCAF1 as an adaptor. The Journal of biological chemistry 282:27046-27057.

Yurkovetskiy, L., M.H. Guney, K. Kim, S.L. Goh, S. McCauley, A. Dauphin, W.E. Diehl, and J. Luban. 2018. Primate immunodeficiency virus proteins $\mathrm{Vpx}$ and $\mathrm{Vpr}$ counteract transcriptional repression of proviruses by the HUSH complex. Nat Microbiol 3:1354-1361.

Zahoor, M.A., G. Xue, H. Sato, and Y. Aida. 2015. Genome-wide transcriptional profiling reveals that HIV$1 \mathrm{Vpr}$ differentially regulates interferon-stimulated genes in human monocyte-derived dendritic cells. Virus research 208:156-163.

Zahoor, M.A., G. Xue, H. Sato, T. Murakami, S.N. Takeshima, and Y. Aida. 2014. HIV-1 Vpr induces interferon-stimulated genes in human monocyte-derived macrophages. PloS one 9:e106418.

Zhang, L., Q. Jiang, G. Li, J. Jeffrey, G.I. Kovalev, and L. Su. 2011. Efficient infection, activation, and impairment of pDCs in the BM and peripheral lymphoid organs during early HIV-1 infection in humanized rag2(-)/(-)gamma C(-)/(-) mice in vivo. Blood 117:6184-6192.

Zhou, X., M. DeLucia, and J. Ahn. 2016. SLX4-SLX1 Protein-independent Down-regulation of MUS81EME1 Protein by HIV-1 Viral Protein R (Vpr). J Biol Chem 291:16936-16947. 


\section{Figure Legends}

Figure 1. Vpr inhibits IFN-I induction in pDCs to enhance HIV-1 replication in co-cultured

CD4+ T cells. (A) HIV -1 or HIV $-1 \Delta$ Vpr viruses infect and replicate in activated CD4+ T cells at similar levels. HIV p24+ cells or supernatant p14 wre measured by flow cytometry or ELISA at 48h post- infection. (B) Schematic representation of HIV-infected T cells and pDC co-culture design. HIV-infected T cells for 48 hours were mixed with pDCs. IFN-I expression and HIV-1 replication in supernatants are measured $24 \mathrm{~h}$ after co-culture. (C) CD4+ T cells infected with HIV1 or HIV-1 $\Delta$ Vpr for 48 hours were co-cultured with or without purified autologous pDCs. HIV-1 replication was measured by p24 ELISA at 24 hour after co-culture. (D) Relative inhibition of replication of HIV-1 or HIV-1 $\Delta$ Vpr in CD4+ T cells by pDCs is summarized with three different donors. (E) IFN- $\alpha$ in the supernatant was measured at 24 hours after co-culture by ELISA. (F) Isotype control or $\alpha$-IFNAR1 antibody was added at the beginning of co-culture, and HIV-1 replication (p24) was measured at 24 hour after co-culture. Error bars represent \pm SD of triplicate experiments. $* * *$ and $* * *$ indicate $\mathrm{p}<0.05,<0.01$ and $<0.001$, respectively.

\section{Figure 2. HIV-infected T cells activate pDCs via CD4-dependent but RT-independent} mechanisms. (A) HIV-1 or HIV-1 $\mathrm{Vpr}$ infected CD4+ T cells were cocultured with or without pDCs, with sCD4 at $20 \mu \mathrm{g} / \mathrm{ml}, 10 \mu \mathrm{g} / \mathrm{ml}$ or $2 \mu \mathrm{g} / \mathrm{ml}$ was added at the beginning of coculture. IFN$\alpha$ in supernatants was measured at 24 hours after co-culture by ELISA. (B) p24 in supernatants was measured at 24 hours after co-culture by ELISA. (C) Activated primary CD4+ T-cells were infected with HIV-1 (MOI = 1) for $48 \mathrm{~h}$. at the same time with viral infection. RT inhibitors nevirapine is $5 \mu \mathrm{m}$. p24 levels in the supernatants were quantified by ELISA. (D) HIV-1 or HIV$1 \Delta \mathrm{Vpr}$ infected CD4+ $\mathrm{T}$ cells were cocultured with or without $\mathrm{pDCs}$. The reverse transcriptase (RT) inhibitor nevirapine (NVP) was added $(5 \mu \mathrm{m})$ at the beginning of coculture. Supernatant IFN- 
$\alpha$ was measured at 24 hours after co-culture by ELISA. (D) Supernatant p24 was measured at 24 hours after co-culture by ELISA. Error bars represent \pm SD of triplicate experiments.

Figure 3. Virion-associated Vpr degrades TET2 to modulate IFN-I induction in pDCs. (A) pDCs were exposed to cell-free HIV-1 or HIV-1 $\Delta$ Vpr viruses for 10 hours, followed with R848 treatment for 16 hours. Supernatant IFN- $\alpha$ was measured by ELISA post R848 treatment. (B) pDCs were exposed to VLP or VLPVPR in the absence or presence of R848. Supernatant IFN- $\alpha$ was measured at 16 hours by ELISA post R848 treatment. VLP or VLPVPR pre-treat pDCs for 10 hours. Error bars represent \pm SD of triplicate experiments. (C) pDCs were exposed to VLPVPR-wT, VLPvPRR80A or VLPVPR-Q65R for 10 hours and treated with R848. Supernatant IFN- $\alpha$ was measured at 16 hours post stimulation. (D) Human pDC-like cell line Gen2.2 cells were transduced with GFPexpressing shRNA control (shCTRL) or shRNA targeting TET2 (shTET2-1 and shTET2-2). TET2 knock-down efficiency was measured by real-time quantitative PCR and western blotting of the protein levels. Two independent TET2 shRNAs both stably reduced TET2 mRNA levels and protein levels in pDC cell line GEN2-2 (Fig. S3). GEN2-2 cells with shCTRL, shTET2-1 and shTET2-2 were exposed to R848 and VLPvPR-wT, VLPvPR-R80A or VLPvPr-Q65R. Supernatant IFN- $\alpha$ was measured at 24 hours post treatment. Error bars represent \pm SD of triplicate experiments. ** and $* * *$ indicate $\mathrm{p}<0.01$ and $\mathrm{p}<0.001$, respectively.

Figure 4. Vpr reduces IRF7 expression via TET2-dependent mechanisms in pDCs. (A) Primary pDCs were treated with VLPvPR-WT, VLPVPR-R80A and VLPVPR-Q65R for 10 hours. Endogenous MyD88, IRAK-1/4, IRF7 and actin proteins were detected by Western blots. Primary pDCs were from three different donors were mixed for WB detection. (B) Primary pDCs were treated with mock, VLP, VLPVPR-wT, VLPVPR-R80A and VLPVPR-Q65R. IRF7 mRNA was measured at 10 hours after treatment. Error bars represent \pm SD of triplicate experiments from three different 
donors. (C) GEN2-2 cells with shCTRL, shTET2-1 and shTET2-2 were treated with VLPVPR-wT, VLPVPR-R80A and VLPVPR-Q65R. IRF7 mRNA was measured at 24 hours after treatment. Error bars represent $\pm \mathrm{SD}$ of triplicate experiments.

\section{Figure 5. Vpr modulates IRF7 expression in pDCs via CXXC5/TET2-dependent} demethylation of the IRF7 promoter. (A) Human pDC-like cell line Gen2.2 cells were transduced with control shRNA (shCTRL) or shRNA targeting CXXC5 (shCXXC5). CXXC5 knock-down efficiency was measured by real-time quantitative PCR. (B) ChIP assays were performed using antibodies against $\mathrm{CXXC5}$ or isotype control $\mathrm{IgG}$, followed by qPCR analysis of IRF7 promoter in shCTRL and shCXXC5 Gen2.2 cells. (C) ChIP assays were performed using antibodies against TET2 or isotype control IgG, followed by qPCR analysis of IRF7 promoter in shCTRL and shCXXC5-transduced Gen2.2 cells. (D) ChIP assays were performed using antibodies against $5 \mathrm{hmC}$ or isotype control $\mathrm{IgG}$, followed by qPCR analysis of IRF7 promoter in shCTRL- and shCXXC5-Gen2.2 cells. (E) ChIP assays were performed using antibodies against $5 \mathrm{hmC}$ or isotype control $\mathrm{IgG}$, followed by qPCR analysis of IRF7 promoter in the presence of VLPvPR-wT, VLPVPR-R80A or VLPVPR-Q65R. ChIP DNA level in each experiment was normalized to the IgG isotype control level (defined as 1). Error bars are derived from three technical replicates. (F) Schematic diagram of Vpr-mediated suppression of IRF7/IFN-I in pDCs to facilitate HIV-1 replication in CD4+ T cells. Upon endocytosis of HIV-1 virions in pDCs, the Vpr protein binds VprBP to target TET2 for degradation, leading to impaired demethylation of the IRF7 promoter and reduced IRF7 expression in pDCs. Reduction of IRF7 and IFN-I by Vpr in pDCs will facilitate HIV-1 replication in CD4+ T cells and other HIV-1 target cells. 


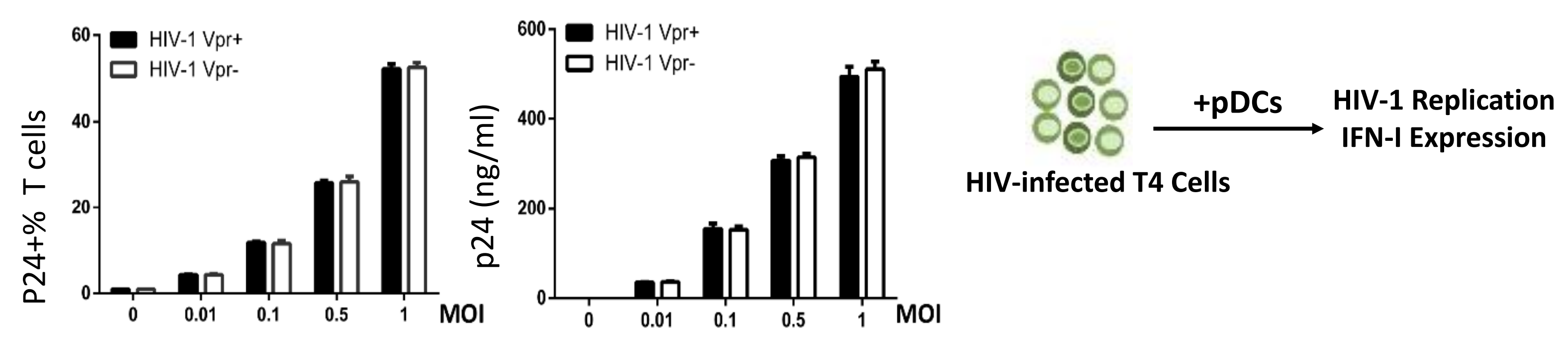

C

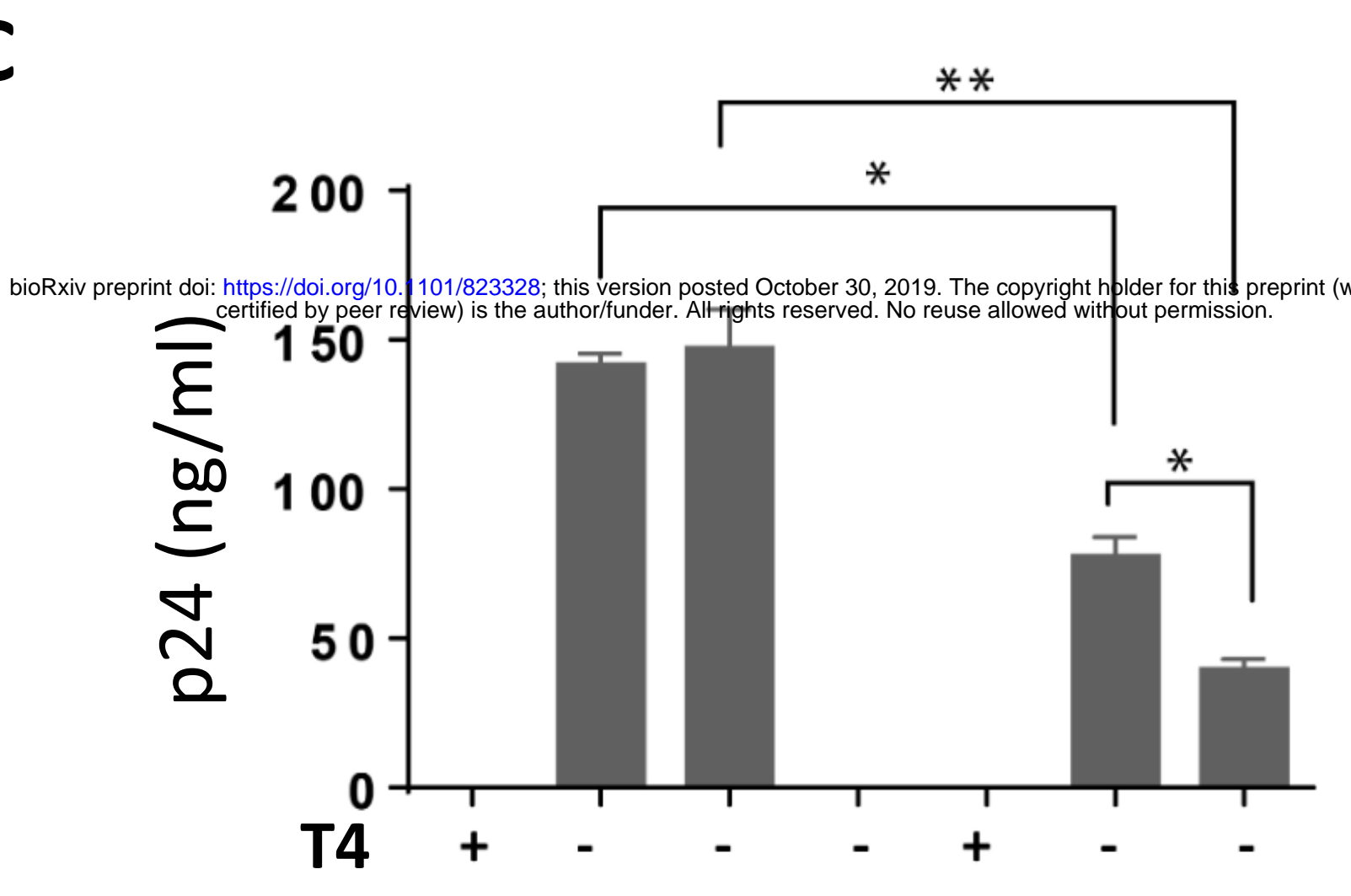

D

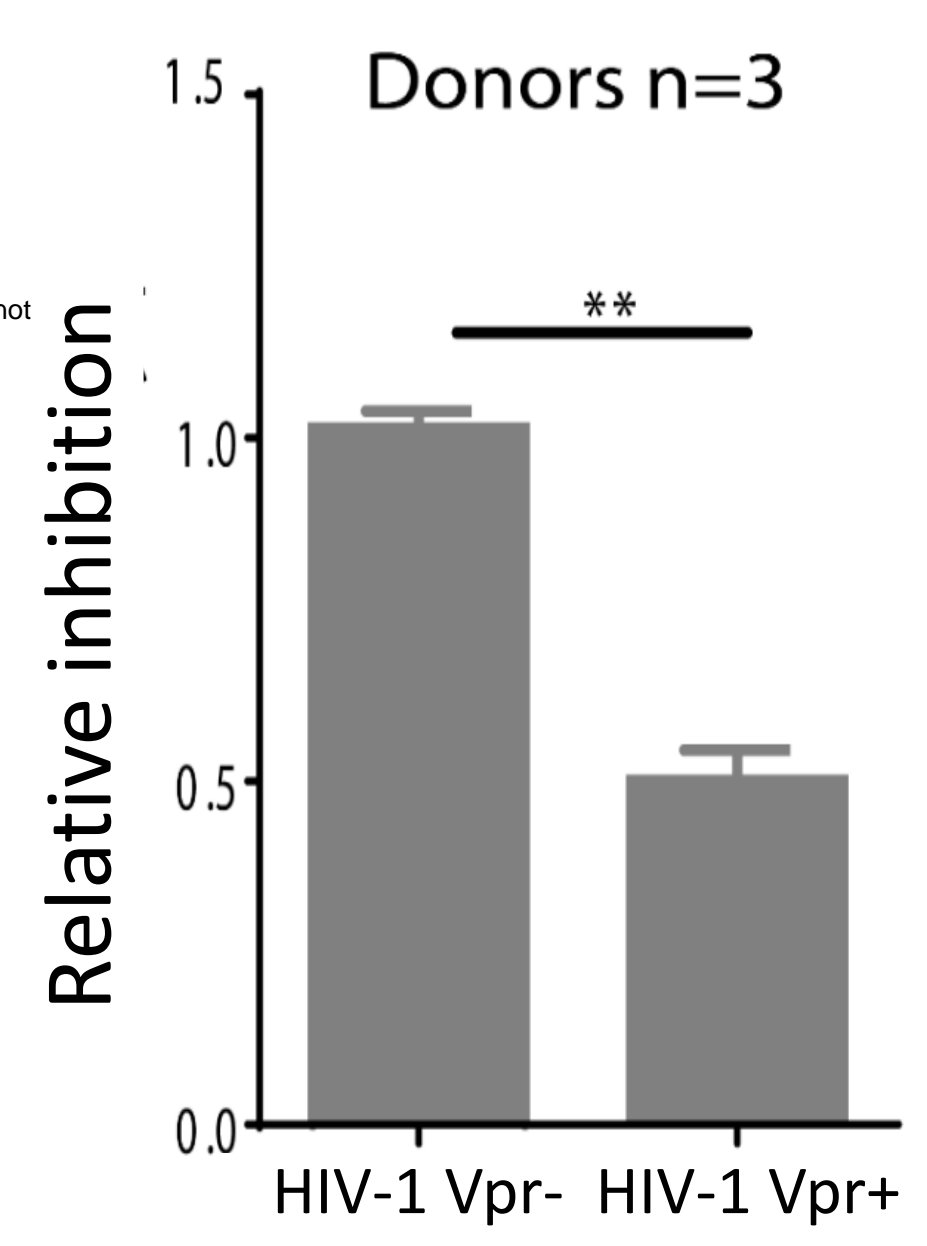

T4+HIV-1 Vpr+

T4+HIV-1 Vpr - - - $+--_{-}+$

HIV-1 Vpr- HIV-1 Vpr+
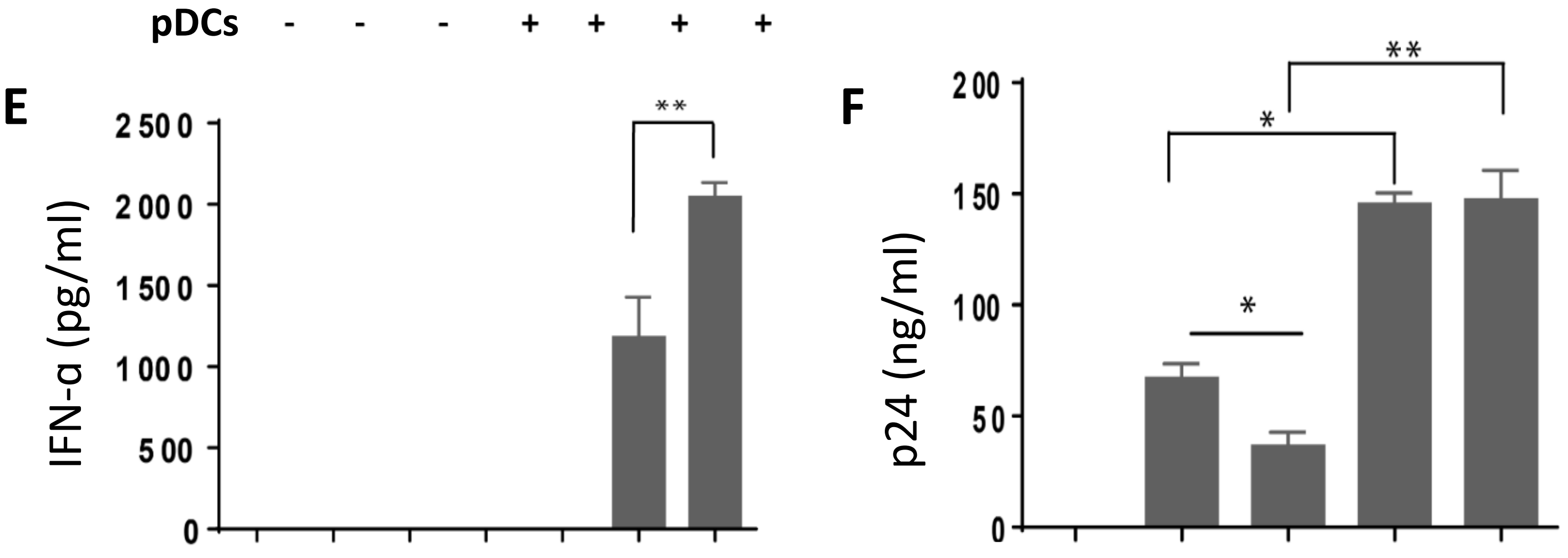

T4

$\mathbf{T}$ T4+HIV-1 Vpr - - - $+--_{-}+$ pDCs - - - ++++

T4+HIV-1 Vpr+ -

T4+HIV-1 Vpr - -

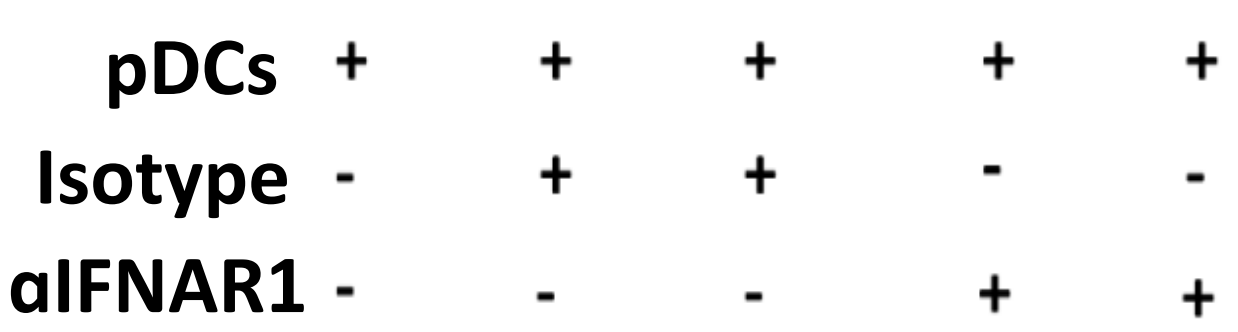




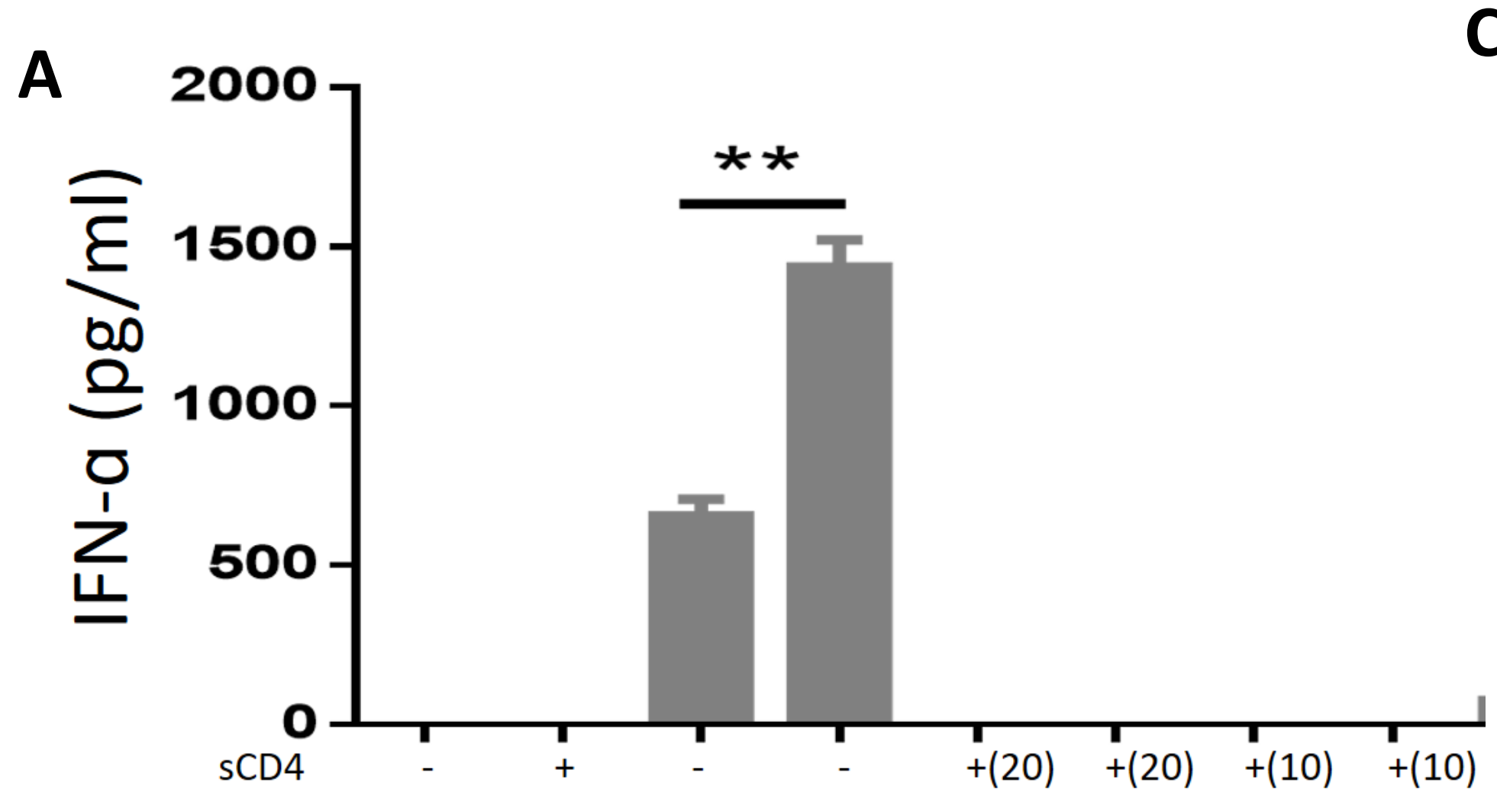

T4+ HIV-1 Vpr+

T4+ bioRxiYgreprint doi: https://doi.org/10.1101/823328; this version posted October 30, 2019. The copyright holder for this preprint (which was not

pDCs $\quad+\quad+\quad+\quad+\quad+\quad+\quad+\quad+\quad+\quad+4$

B

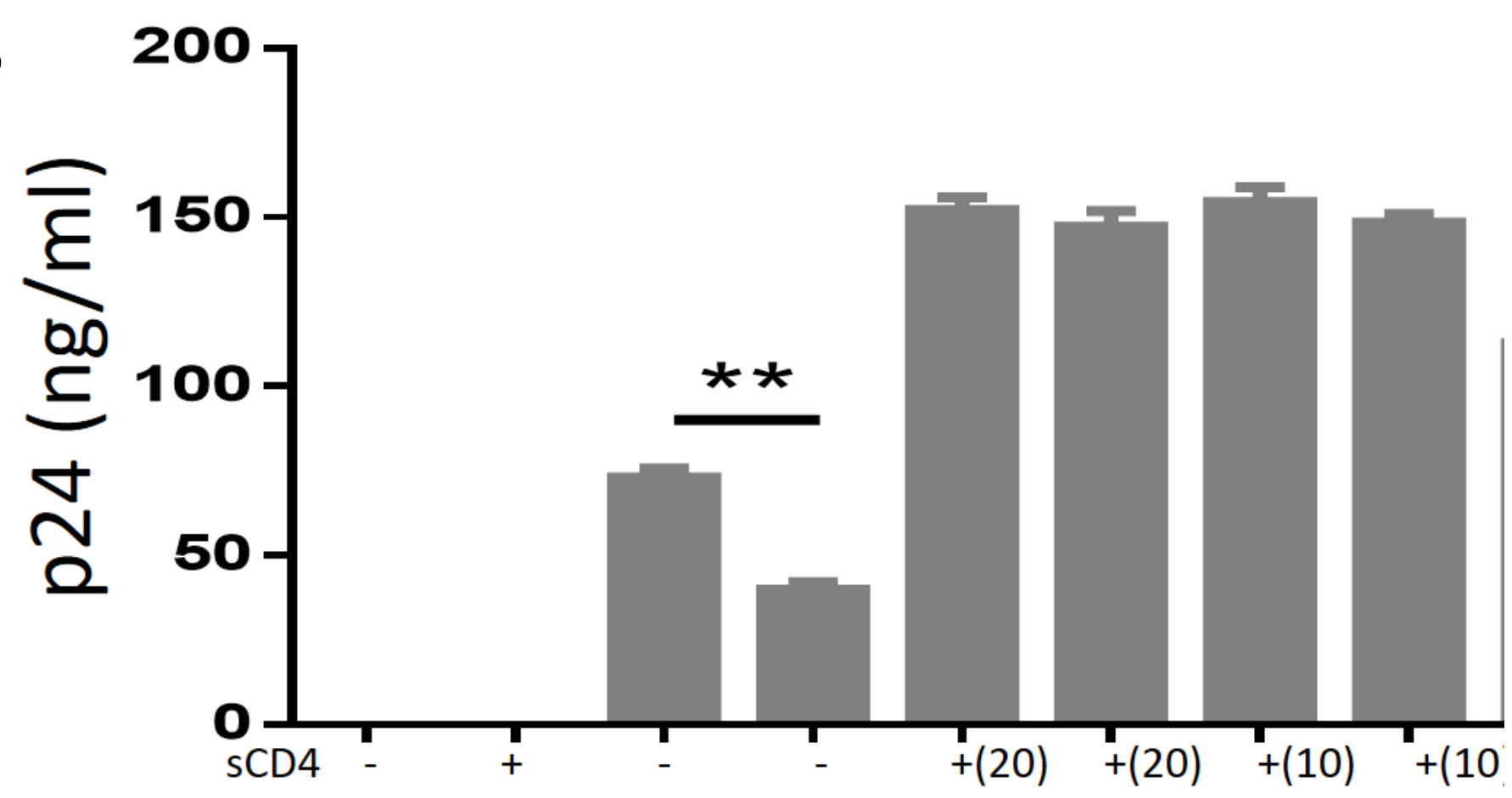

T4+ HIV-1 Vpr+

T4+ HIV-1 Vpr-

pDCs +
C

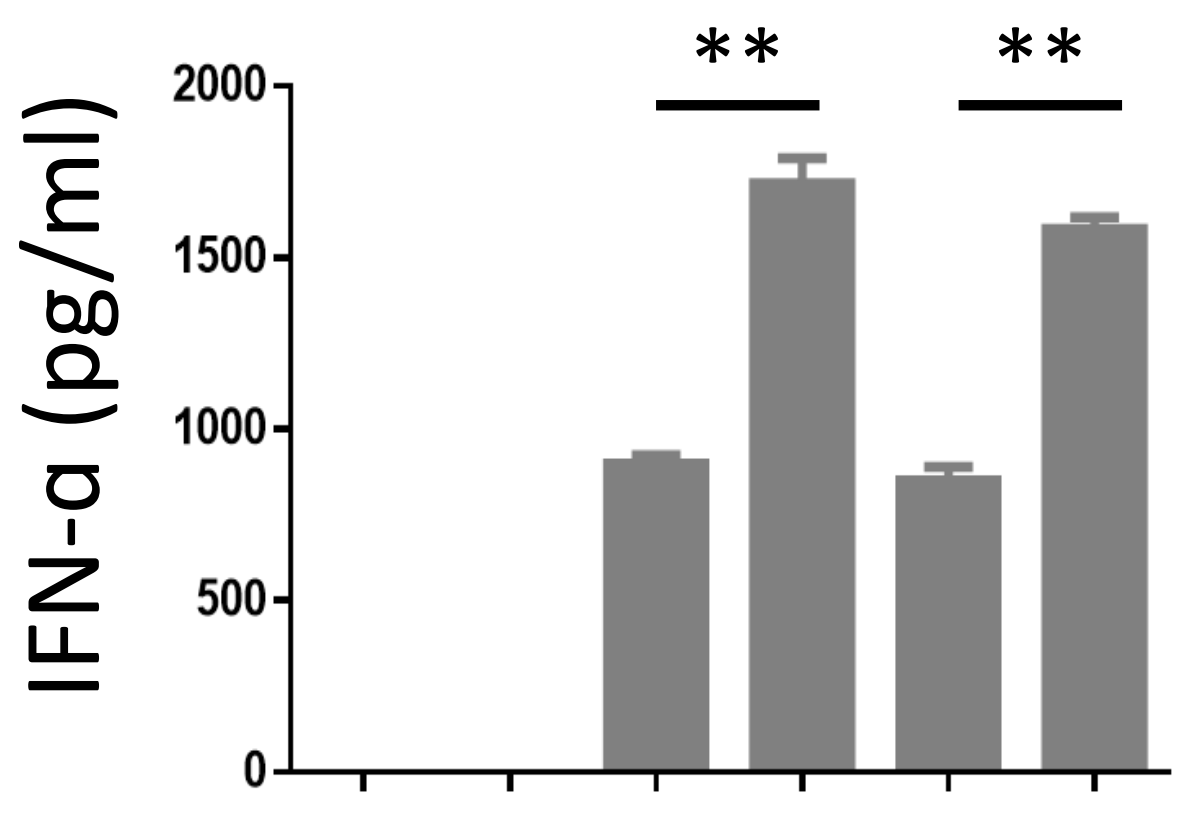

RT inhibitor T4+ HIV-1 Vpr+

T4+ HIV-1 Vpr-

pDCs

D

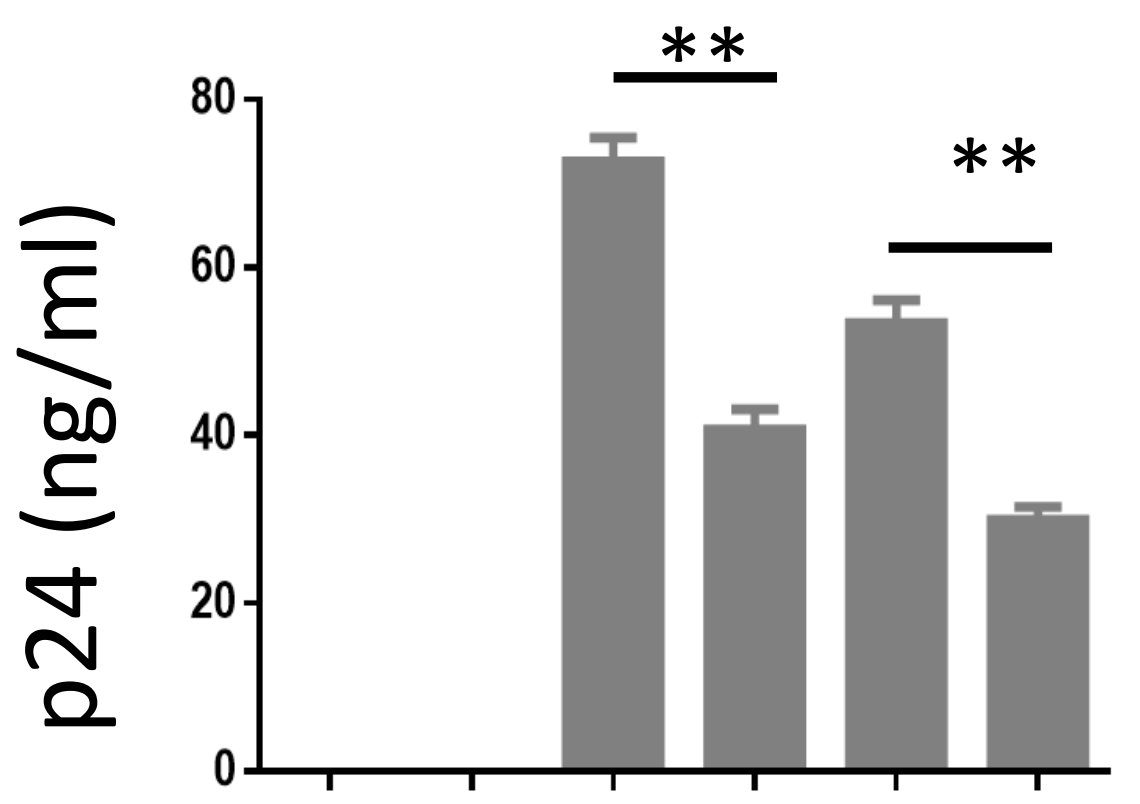

RT inhibitor

T4+ HIV-1 Vpr

T4+ HIV-1 Vpr-

pDCs 
A

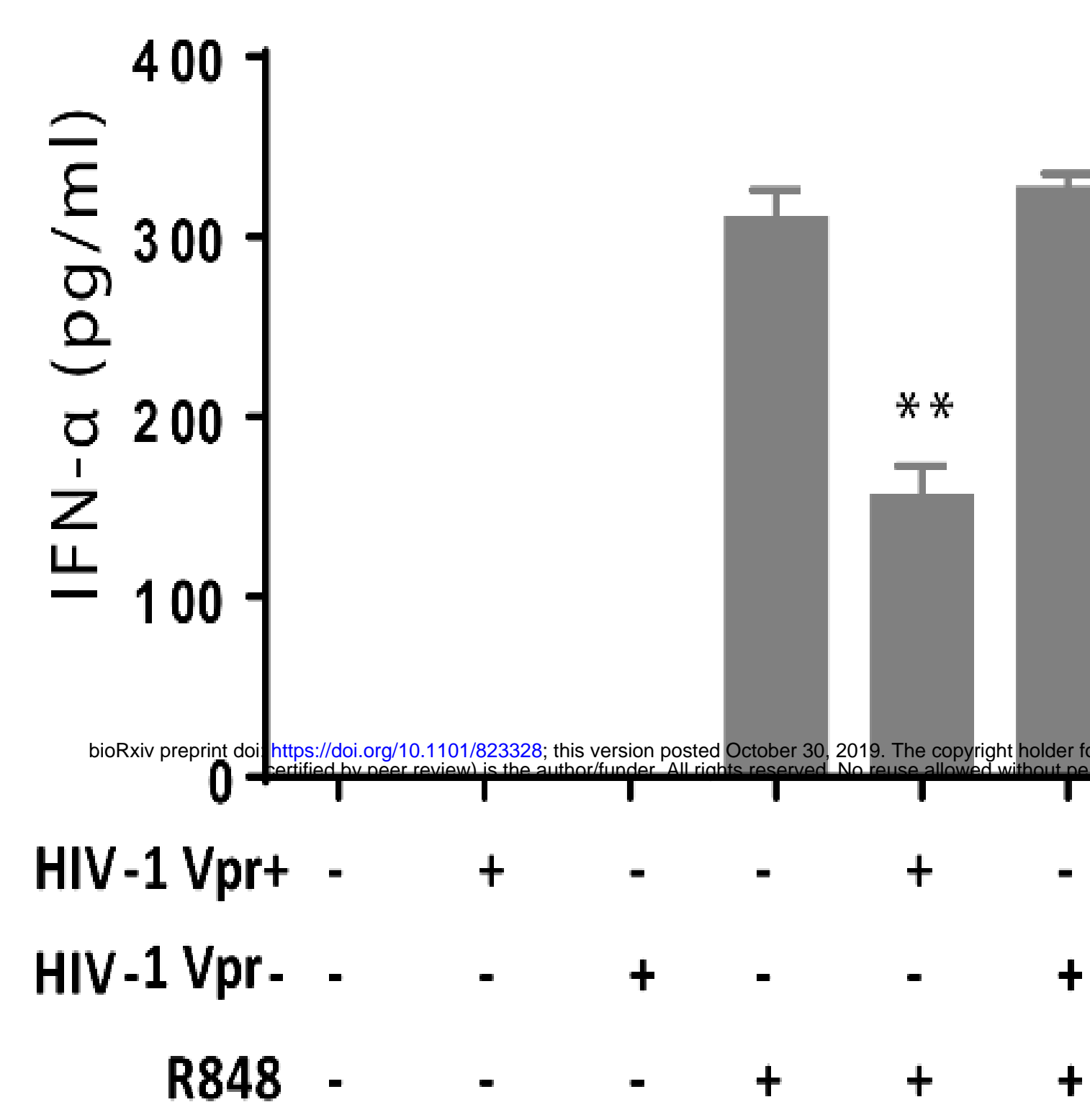

B

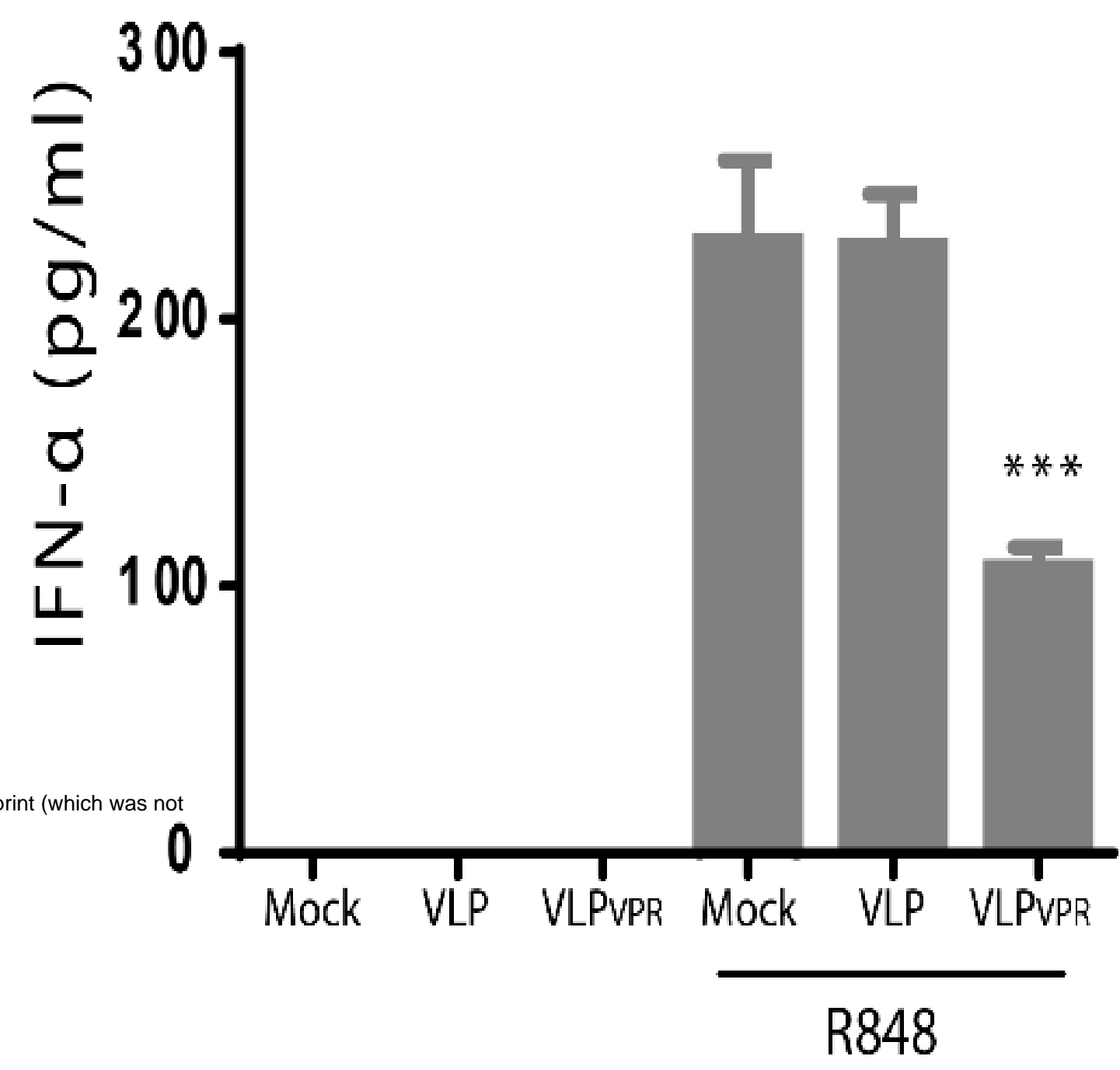

D

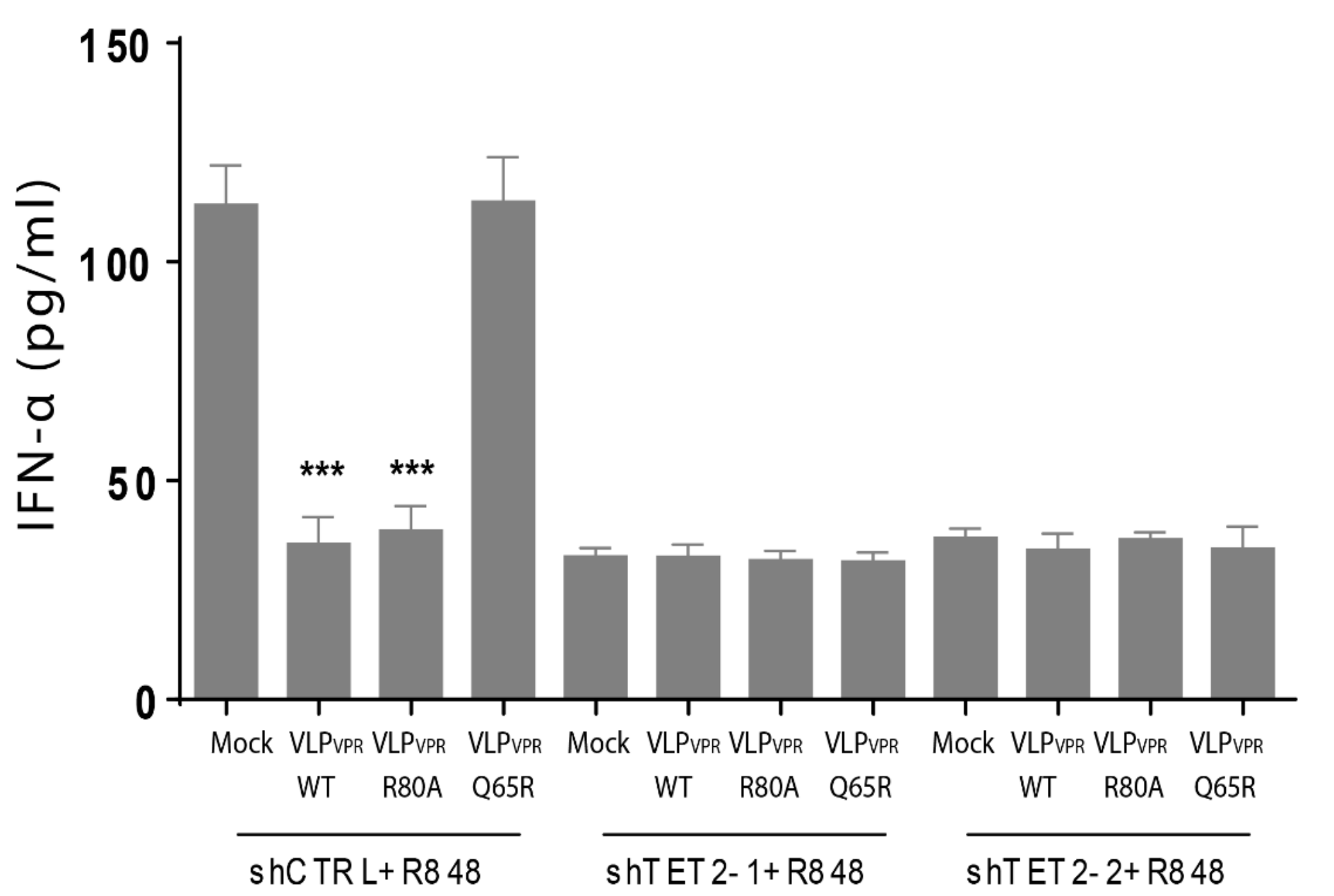




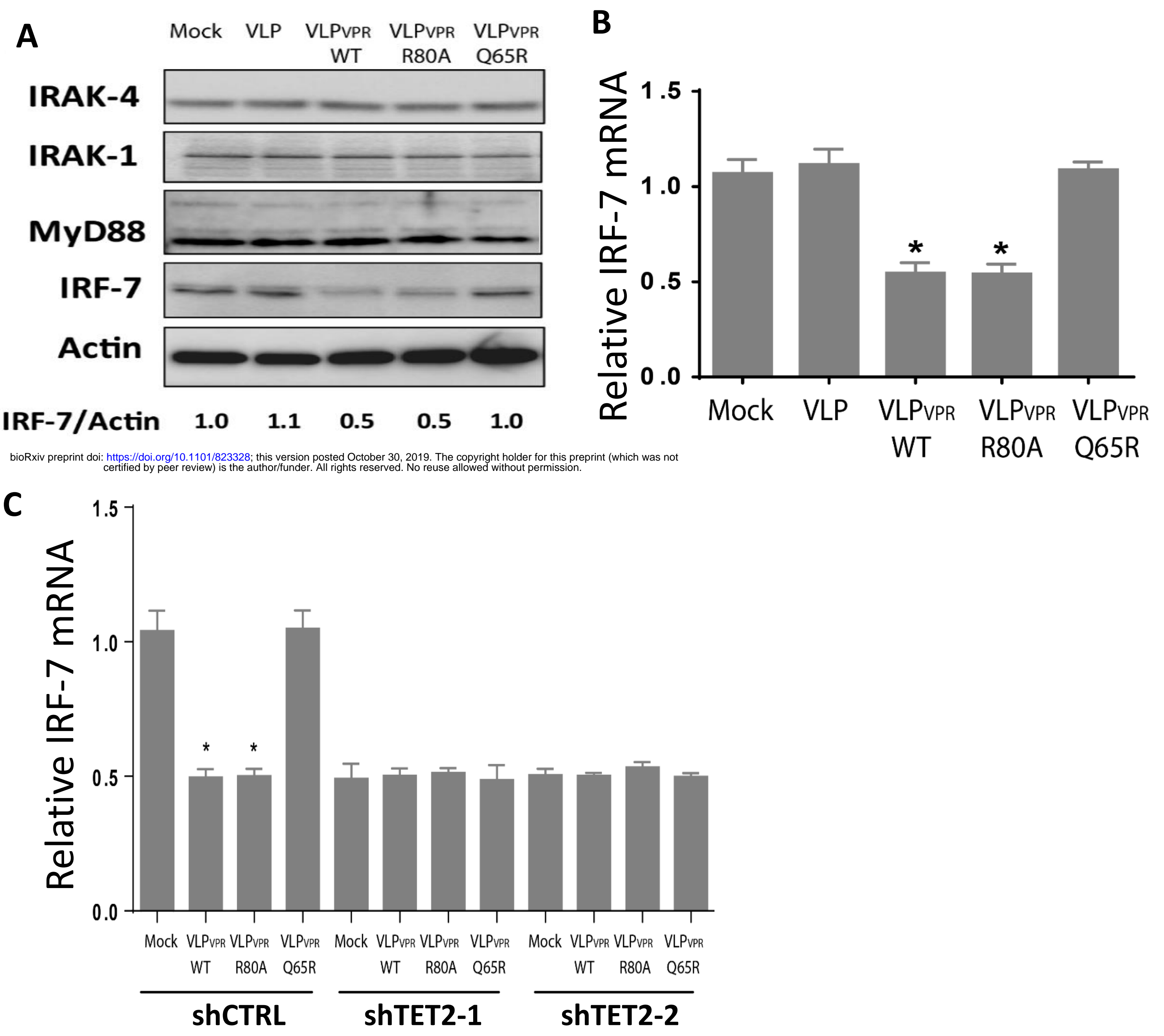


A

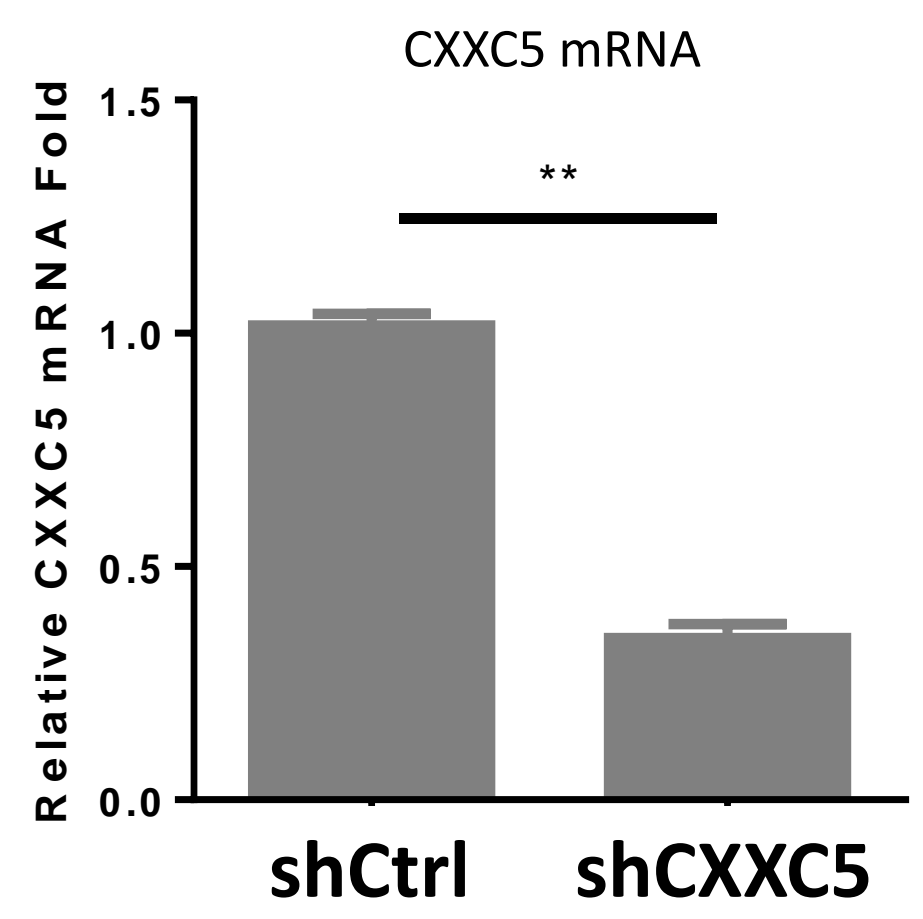

B

CXXC5

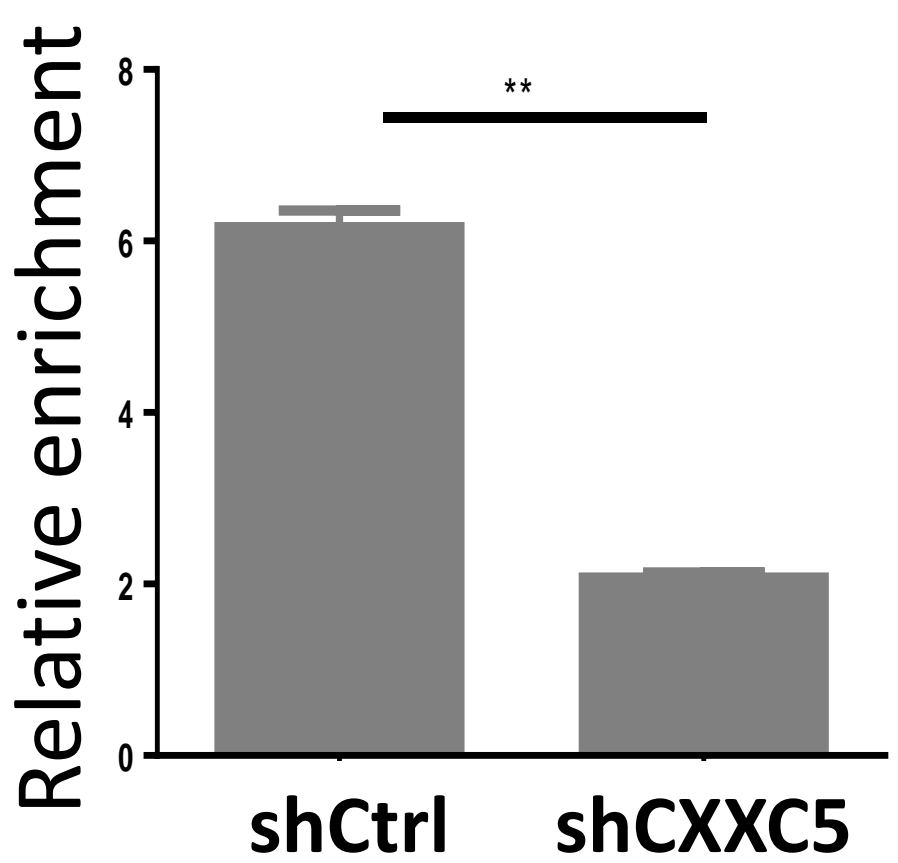

C
D

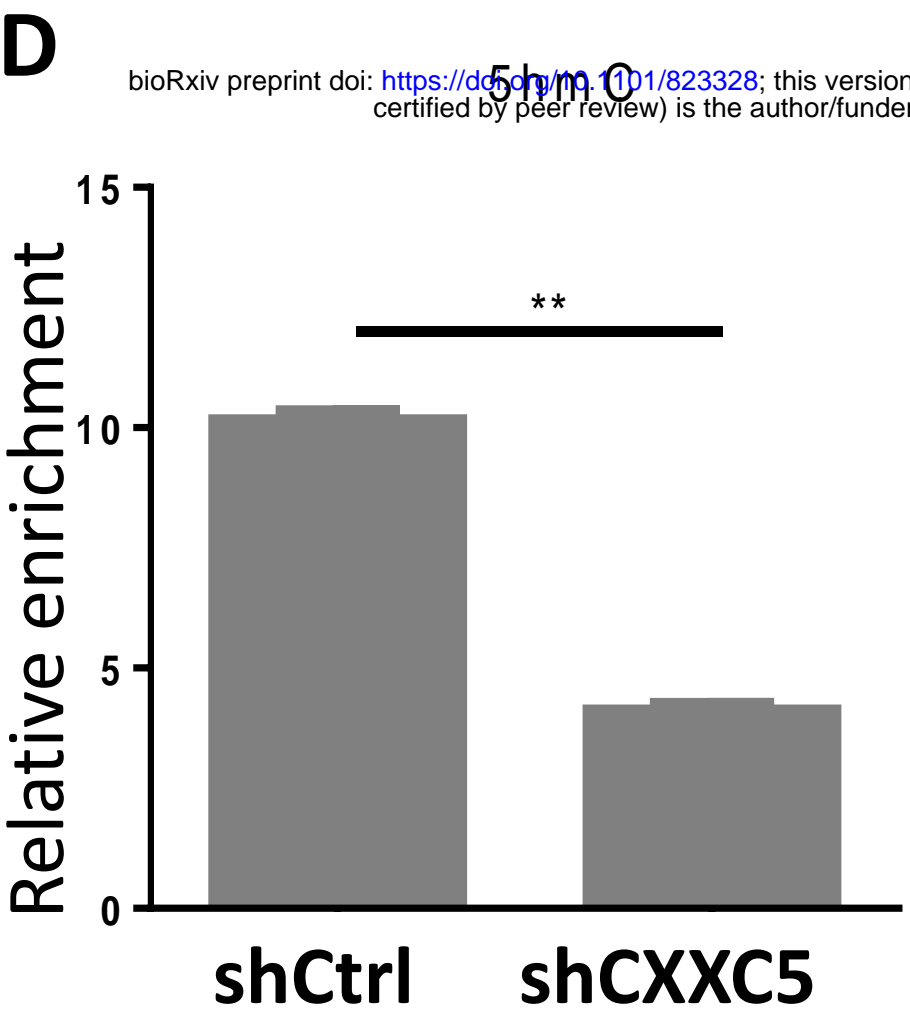

$\mathbf{E}_{2}$

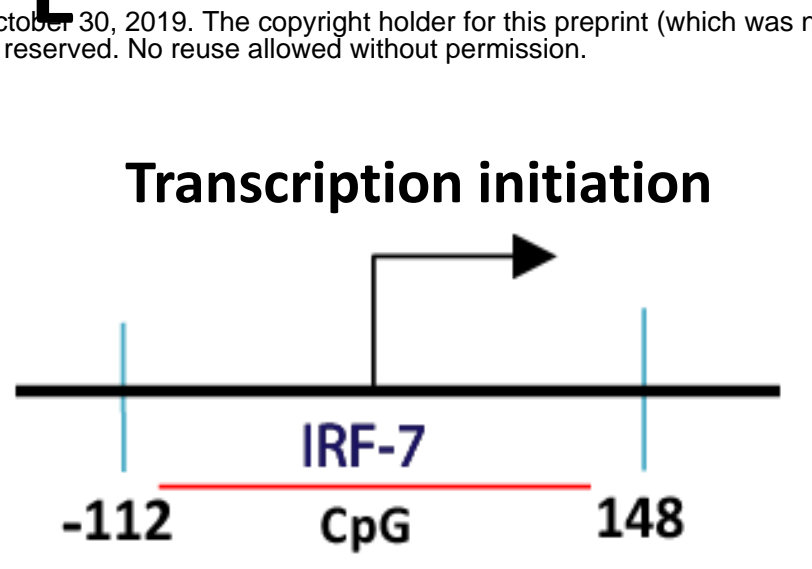

$\mathbf{F}$
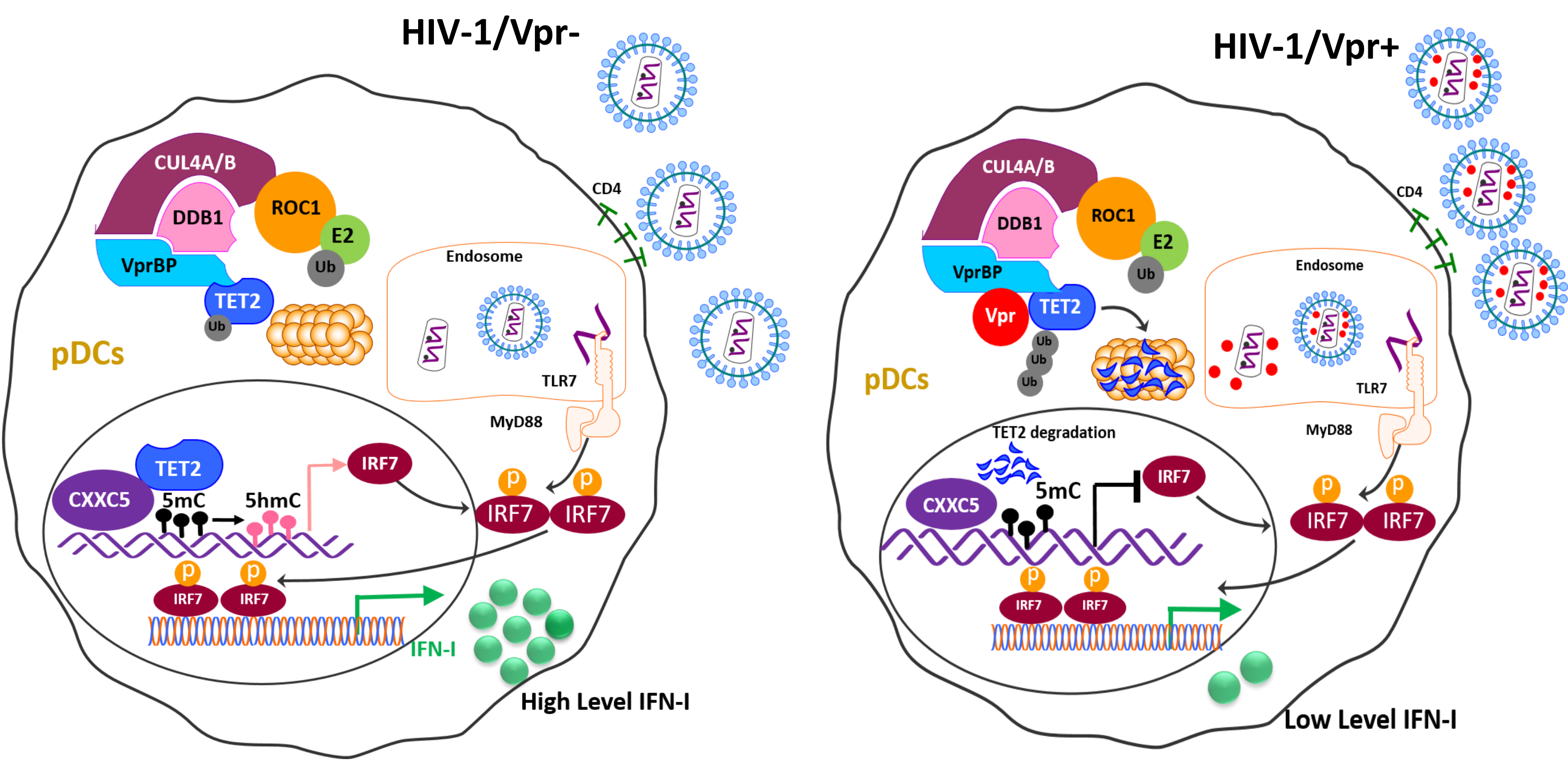